\title{
Hydrogeologische Herkunft und geothermisches Potenzial der Thermalquellen von Hintertux, Österreich
}

\author{
Claus-Dieter Heldmann ${ }^{1,2} \cdot$ Ingo Sass ${ }^{2,3} \cdot$ Rafael Schäffer $^{2}$ \\ Eingegangen: 13. Mai 2019 / Überarbeitet: 17. November 2019/ Online publiziert: 25. März 2020 \\ (c) Der/die Autor(en) 2020
}

\section{Zusammenfassung}

Europas höchste Thermalquellen in Hintertux, Tirol, sind bis zu $22,5^{\circ} \mathrm{C}$ warm und werden zu etwa $30 \%$ genutzt. Bisher lag kein detailliertes konzeptionelles Modell vor, das alle Besonderheiten der Anomalie gemeinsam erklärt hätte. Nur mit einem hydrogeologischen Modell ist eine Erweiterung der Nutzung nachhaltig planbar. Es wurden klassische hydrogeologische und hydrochemische Methoden eingesetzt, unter anderem natürliche Tracer, geochemische Indikatoren und stabile Isotopen $\left(\delta^{18} \mathrm{O}, \delta \mathrm{D}, \delta^{34} \mathrm{~S}\right)$, um die Entstehung der Thermalwässer und darin involvierte Grundwasserleiter zu identifizieren. Die Untersuchungen wurden 2011-2018 an Wässern aus 14 weiteren Quellen und zwei Tunneln durchgeführt. Die Studie zeigt, dass das Grierkar zum meteorischen Einzugsgebiet der Thermalquellen gehört, wobei der Abfluss über die Schwinden an der Grieralm in den Hochstegenmarmor gelangt. Die Temperaturerhöhung erfolgt ursächlich aus dem weiteren Zufluss (20-40\%) von tieferen Zentralgneiswässern im Nordhang des Schmittenberges, wobei diese hydraulische Verbindung wahrscheinlich im Zusammenhang mit den Tuxer Scherzonen steht.

\section{Hydrogeological genesis and geothermal potential of the thermal springs of Hintertux, Austria}

\begin{abstract}
Europe's highest thermal springs are located at $1500 \mathrm{~m}$ asl in Hintertux, Tyrol. Only $30 \%$ of the springs with temperatures up to $22.5^{\circ} \mathrm{C}$ are utilized. Future exploration and exploitation will require quantifying their resource potential and development of a conceptual hydrogeological model. A detailed and comprehensible explanation of the thermal anomaly has not yet been formulated. Methods including a combination of natural tracers, geochemical indicators and stable isotopes $\left(\delta^{18} \mathrm{O}\right.$, $\delta \mathrm{D}, \delta^{34} \mathrm{~S}$ ) have been used for identifying their genesis and their associated aquifers. Hydrogeological and hydrochemical investigations have been conducted from 2011-2018, evaluating groundwater from 14 surrounding springs and two tunnels. This study shows that the thermal water originates mainly from rock glaciers and meteoric waters from the Grierkar, entering swallow holes of the Hochstegen formation at the Grieralm. The thermal anomaly is caused by a groundwater component (20-40\%) from footwall gneisses, probably connected to the marble aquifer by the Tux shear zones.
\end{abstract}

Keywords Thermal water $\cdot$ Tux Shear Zones $\cdot$ Hochstegen formation · Tauern Window

Claus-Dieter Heldmann

claus-dieter.heldmann@afry.com

1 Pöyry Austria GmbH, Strubergasse 30, 5020 Salzburg, Österreich

2 Fachgebiet Angewandte Geothermie, Institut für Angewandte Geowissenschaften, Technische Universität Darmstadt, Schnittspahnstraße 9, 64287 Darmstadt, Deutschland

3 Darmstädter Exzellenz-Graduiertenschule für Energiewissenschaft und Energietechnik, Technische Universität Darmstadt, Otto-Berndt-Str. 3, 64287 Darmstadt, Deutschland

\section{Einleitung}

\section{Hintertuxer Thermalquellen}

Die mit maximal etwa $23^{\circ} \mathrm{C}$ akratotherme Quellgruppe in

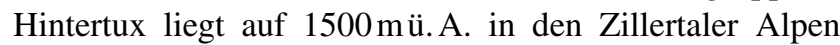
(Abb. 1) und ist damit die höchst gelegene Thermalquelle Europas (Carlé 1975). Die Thermalquellen wurden bereits um 1600 von Burglechner beschrieben (Elster et al. 2018) und sind seit 1847 im Privatbesitz der Familie, die das Vorkommen heute balneologisch nutzt. Bisherige Veröffentlichungen (Elster et al. 2018; Zötl und Goldbrunner 


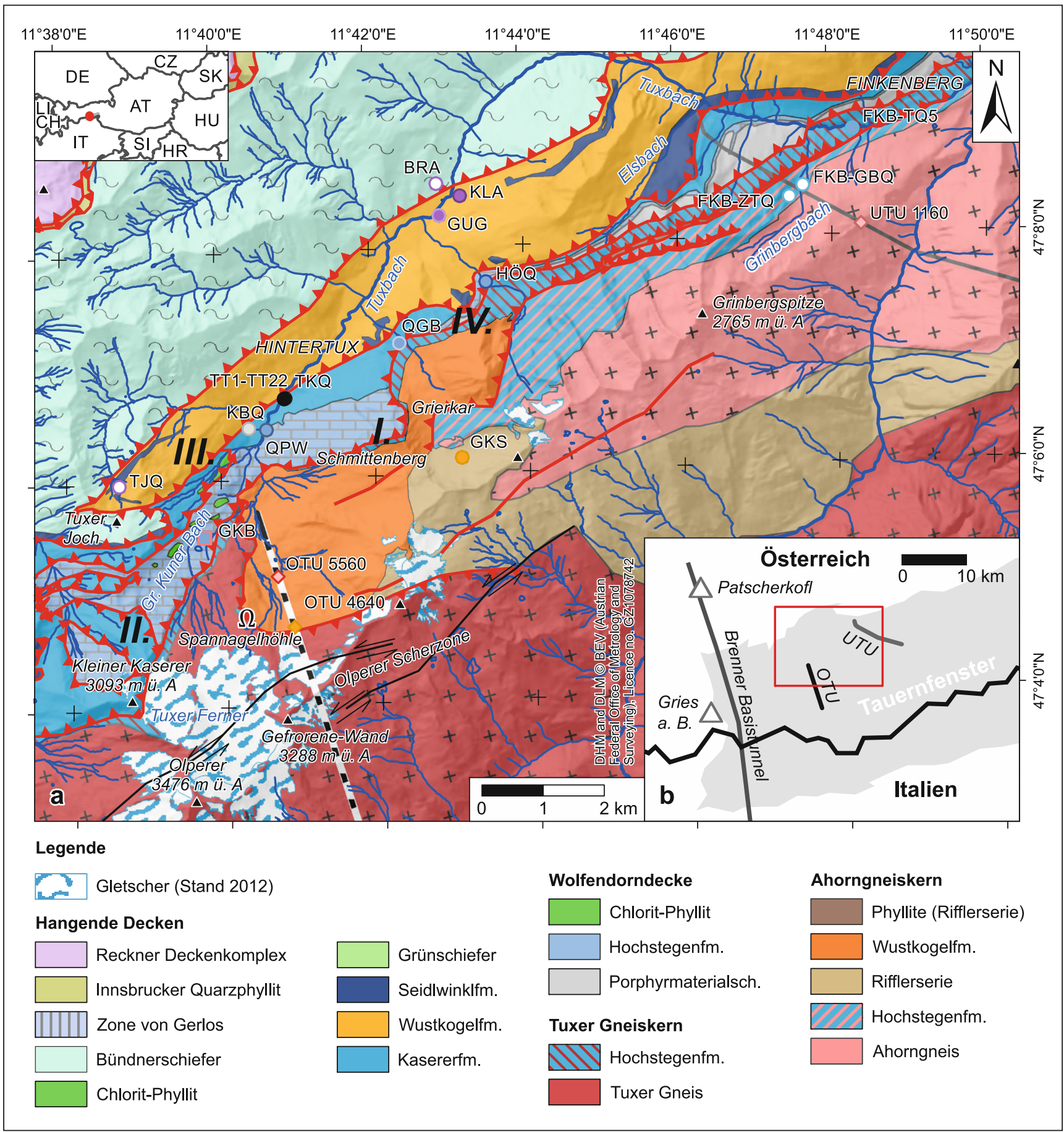

Abb. 1 Abgedeckte geologische Karte des Tuxertals (GBA 2011; verändert) ohne Quartär mit den Lokationen der Thermalquellen (TT) und weiterer Probennahmestellen

Fig. 1 Geological map of the Tux Valley (modified from GBA (2011), Quaternary units removed) with the Tux Thermal Springs and other sampling locations

2013; Carlé 1975) machen unterschiedliche Angaben zur Anzahl und Benennung der Quellaustritte, zu Temperaturen und Schüttungen. Auch sind die hydrochemischen Analysen nicht einheitlich. Zumeist beziehen sich die Angaben auf die zwei wasserrechtlich zur Nutzung genehmigten
Quellaustritte. Bei der ältesten vorliegenden Untersuchung von 1885 von Kauer (Carlé 1975 ) werden $41 / \mathrm{s}$ bei $22,5^{\circ} \mathrm{C}$ angegeben. Die Analyse wird hier wegen mangelnder Vergleichbarkeit und Genauigkeit der Methoden mit heutigen Analysen nicht widergegeben. 1952 untersuchten Schminz- 
ky, Komma und Mutschlechner (Czurda 1993) 22 Quellaustritte, die insgesamt $15-201 / \mathrm{s}$ schütteten, bei einer Wassertemperatur von $11,8-22,2^{\circ} \mathrm{C}$ (Tab. 1). Bis zu der hier vorliegenden Studie war dies die einzige komplette Aufnahme aller Austritte; zu den publizierten Daten gehörten auch Messungen von Radon und Uran. Begleitend zum Bau des Tunnels zur oberen Tuxbachüberleitung wurde 1969-1974 eine Messreihe mit wöchentlichen bis monatlichen Intervallen an den genutzten Quellen realisiert. Diese Zeitreihe bildet neben dieser Arbeit die einzige Erfassung von Jahresgängen. Für 1993 wird die Schüttung der gesamten Quellgruppe mit 15-201/s angegeben (Elster et al. 2018).

Zur Erklärung der erhöhten Temperatur gibt es verschiedene Hypothesen, davon seien hier drei gegeben:

1. Mutschlechner (vgl. Carlé 1975) vermutet 1966 eine tiefe Versickerung im Kristallin am gletscherbedeckten Tuxer Hauptkamm und Transport durch sich kreuzende Störungssysteme bis zum Austritt in den Schuttfächer im Bereich der Quellen.

2. Czurda (1993) vermutet den Ursprung der Quellen in einer tektonischen Grenze, sieht aber aufgrund der hohen Schüttung ein Karstsystem als Aquifer (wobei er die Phyllite der Kasererserie im Liegenden des Schuttfächers allerdings der Hochstegenformation zurechnet); er stellt dabei zu Recht fest, dass die Temperatur im Widerspruch steht zur Charakteristik von Karstquellen und dass die auf den Gneisen hangenden Carbonate mit einer ortsüblichen geothermischen Tiefenstufe hangwärts nicht tief genug reichen. Czurda (1993) kommt daher zu dem Schluss, dass die Wässer unter Hintertux im Hochstegenmarmor aufsteigen, ohne den notwendigen Mechanismus - dichtegetriebene Konvektion oder Arteser, Gaslift ist auszuschließen - näher zu diskutieren.

3. Elster et al. (2018) äußern eine ähnliche Vermutung und postulieren hinsichtlich der Urangehalte eine Herkunft entweder aus den Zentralgneisen oder aber den hangenden permischen Metasedimenten, räumen aber ein, dass diese nicht zugleich der Ursprung der Temperaturerhöhung sein muss und verweisen alternativ auf eine tiefere Zirkulation (also unterhalb $1500 \mathrm{~m}$ ü. A.) im Marmorkarst der Hochstegenformation.

In der bisher veröffentlichten Literatur bleibt das eigentliche Einzugsgebiet der Thermalquellen in Hintertux unscharf. Die Gesamtschüttung der Hintertuxer Thermalquellen ist für eine Quellgruppe südlich des Tuxbaches auffällig hoch, denn höhere Schüttungen erreichen nur die Gruppen der Klausbodenquellen, der Guggerquellen sowie der Tuxbachquelle (Abb. 1, Tab. 2) und diese stehen dabei mutmaßlich in Zusammenhang mit Karstaquiferen - erstere mit dem des Triasdolomits bzw. letztere mit dem des Hochstegenmarmors (Sass und Schäffer 2018). Eine Verbindung zu lokalen verkarstungsfähigen Carbonatformationen ist für

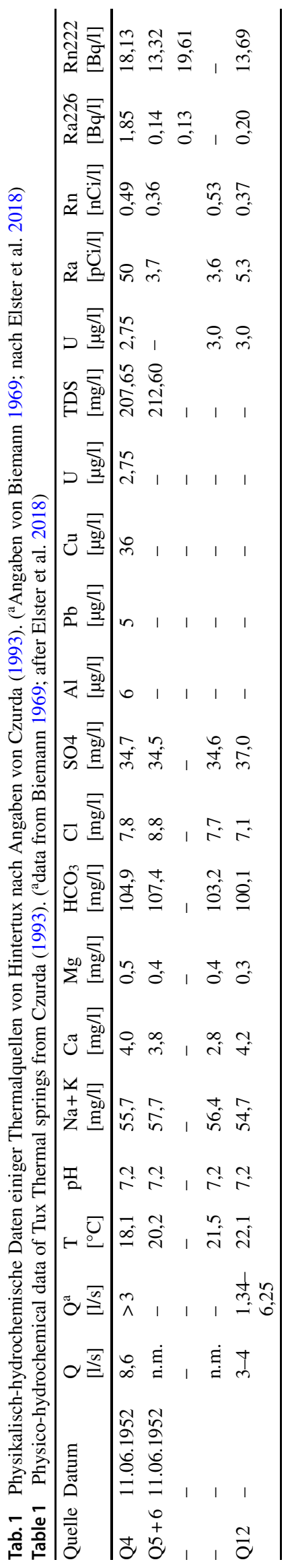


mehrere Autoren naheliegend, allerdings blieb der Mechanismus bis dato ungeklärt.

\section{Hydrogeologie und Carbonatkarst im Tuxertal}

Das hochalpine Tuxertal ist im Bereich von Hintertux (1500mü.A.) von steilen Bergen umgeben wie dem Schmittenberg (2731 mü.A.) im E, die mit dem Olperer am Tuxer Hauptkamm Höhen bis zu 3476 mü. A. erreichen. Die geologische Situation am Nordwestrand des Tauernfensters exponiert mehrere Einheiten steil einfallender Carbonatgesteine. Diese Struktur hat zur Entstehung mehrerer Kilometer langer Höhlensysteme geführt. Die Verkarstung lief sogar während den Stadialen weiter, wie beispielsweise belegt in der Spannagelhöhle $3,5 \mathrm{~km}$ südsüdwestlich von Hintertux (Spötl und Mangini 2010; Cliff et al. 2010) (Abb. 1).

Die Marmore der Hochstegenformation treten durch Deckenstapelung insgesamt in drei Decken übereinander auf:

a) in der Hochstegenzone des Ahorngneiskerns,

b) in der Hülle des Tuxer Gneiskerns und

c) in der Wolfendorndecke (Frisch et al. 1984; Kurz et al. 1998; Schmid et al. 2013).

Dadurch und wegen ihrer Isoklinalfaltung und lokaler Schichtdopplungen durch deckeninterne Überschiebungen sind die Gesamtmächtigkeiten sehr unterschiedlich.

Die Karstphänomene in diesen Marmoren beinhalten neben zahlreichen Höhlen auch Bachschwinden wie diejenigen auf der Grieralm oder die von Schäffer und Sass (2018) auf der Grinbergalm nachgewiesene Schwinde, sowie dmgroße Karströhren mit hohen Grundwassergeschwindigkeiten selbst in mehreren hundert Metern unter Bachniveau (Sass et al. 2016a). Wässer und Quellen aus den Marmoren der Hochstegenformation wurden bereits mehrfach untersucht (Sass et al. 2016b). Die Grundwasserkörper im Liegenden der Carbonate in den Gneiskernen sind Kluftgrundwasserleiter. In einer vorausgegangenen Studie im gleichen Arbeitsgebiet wurden diese untersucht, wobei insbesondere festgestellt wurde, dass sie zu großen Teilen von Gletschern und Blockgletschern gespeist werden und in OstWest-Richtung durch das Tuxer Störungssystem eine ausgeprägte hydraulische Anisotropie durch die hohen Durchlässigkeiten in den fast vertikalen Störungsebenen besitzen (Heldmann et al. 2019).

\section{Motivation und Ziele}

Österreich entwickelt seit einigen Jahren stetig die geothermische Nutzung weiter (Goldbrunner 2010; Neubarth und Kaltschmitt 2013) und die aufgezeigten Möglichkeiten oberflächennaher Geothermie in Österreich zur Bereitstellung von Heizwärme (Götzl et al. 2010) können in Zu- kunft zusätzlich zur Nutzung dieser erneuerbaren Energie und somit zur Emissionsreduktion von Treibhausgasen beitragen. Weltweit gesehen spielen Thermalquellen in der geothermischen Nutzung eine untergeordnete Rolle (Lund 2010), was vermutlich vor allem an ihrem räumlich begrenzten Vorkommen liegt. Trotzdem sind solche Quellen - bei ausreichender Schüttung und Temperatur - als natürliche Ressource über Badezwecke hinaus mittels Wärmepumpen für Wärmegewinnung nutzbar und wirtschaftlich interessant. Trotz der langen Tradition als Badeort werden die Hintertuxer Thermalquellen bisher mit weniger als $30 \%$ der Gesamtschüttung genutzt. Sie könnten aber hinsichtlich des Wärmebedarfs in dem touristisch stark genutzten Gebiet emissionssparend einen größeren Teil des lokalen Energiebedarfs geothermisch decken. Allerdings fehlt ohne Kenntnisse über Genese und Zirkulationspfade des Thermalwassers weiterhin die wichtigste Voraussetzung für eine nachhaltige wirtschaftliche Erschließung. Eine Erkundungsgrabung einige hundert Meter SE bergaufwärts war nicht fündig geworden (mündl. Auskunft der Eigentümer). Drei Ausgangsbedingungen definieren die folgend diskutierten vier möglichen Zirkulationspfade (I-IV in Abb. 1): Die auffälligen Temperaturen, kurzfristige Schüttungssteigerungen in Reaktion auf starke Niederschlagsereignisse und der Ca-carbonatische Charakter der Thermalquellen deuten auf einen - in Anteilen - tiefen Fließpfad durch durchlässige Carbonataquifere hin. Damit sind die nächstgelegenen plausiblen Einzugsgebiete um Hintertux:

I. Grundwasserneubildung in den Gneisen und Marmoren am Schmittenberg (2731 mü. A.) und Transport durch den Hochstegenmarmor.

II. Einzug im verkarsteten Hochstegenmarmor am Kleinen Kaserer (3093 mü. A.) südlich des Tuxer Jochs mit folgendem Transport durch den Aquifer nach NE und (artesischer) Austritt im Schuttfächer.

III. Versickerung in den triassischen Dolomitgesteinen der Modereckdecke auf der nordwestlichen Talseite des Tuxbaches, Absinken noch unter Quellaustrittsniveau bis in Tiefen unterhalb des Ausstreichens der grundwasserhemmenden Kasererserie, dort findet der Übergang in die Wolfendorndecke statt und folgend ein Aufstieg durch den Hochstegenmarmor.

IV. Einzug aus dem Grierkar durch Versickerung in den Schwinden im Hochstegenmarmor auf der Grieralm (1787 mü. A.) mit Erwärmung durch Beiträge aus den Tuxer Scherzonen in den liegenden Gneisen.

Die vorliegende Studie soll über den Stand bisheriger Publikationen hinaus die Einzugsgebiete, die Fließpfade und eine energetische Quantifizierung der Ressource diskutieren. Dabei werden neben allen genannten bisher verfügbaren historischen Zeitreihen eigene langjährige Mes- 
Tab. 2 Liste der untersuchten Quellen und Bäche mit hydrologisch zugehöriger geologischer Einheit

Table 2 List of monitored springs and creeks and their hydrological related geological unit

\begin{tabular}{|c|c|c|c|}
\hline Gruppe & Bezeichnung & Kürzel & Zugeordnete geologische Einheit \\
\hline \multirow[t]{2}{*}{$\begin{array}{l}\text { Quellen im } \\
\text { Bereich Hintertux }\end{array}$} & $\begin{array}{l}\text { Hintertuxer Thermalquellen } \\
\text { (22 Austritte, } 2-10 \mathrm{~m} \text { oberhalb Tuxbach) }\end{array}$ & TT01-TT22 & - \\
\hline & $\begin{array}{l}\text { Tuxer Kalte Quellen } \\
\text { (zwischen Thermalquellen und Tuxbach) }\end{array}$ & TKQ & Hochstegenformation \\
\hline \multirow{3}{*}{$\begin{array}{l}\text { Grundwasseraustritte } \\
\text { in nahegelegenen } \\
\text { Tunneln }\end{array}$} & Obere Tuxbachüberleitung, Station $4640 \mathrm{~m}$ & OTU 4640 & Wustkogelfm./Rifflers \\
\hline & Obere Tuxbachüberleitung, Station $5570 \mathrm{~m}$ & OTU 5570 & Ahorngneis \\
\hline & Untere Tuxbachüberleitung, Station $1160 \mathrm{~m}$ & UTU 1160 & Ahorngneis \\
\hline \multirow{6}{*}{$\begin{array}{l}\text { Gewässer im } \\
\text { Zusammenhang } \\
\text { mit } \\
\text { Hypothesen I-IV }\end{array}$} & Quelle an der Pferdeweide (I, II) & QPW & Hochstegenformation \\
\hline & Großer Kunerbach (II) & GKB & Hochstegenfm. u. a. \\
\hline & Kaiserbründelquelle (III) & KBQ & Kasererformation \\
\hline & Tuxer Joch-Quelle (III) & TJQ & Seidelwinklformation \\
\hline & Grierkarquelle (IV) & GKQ & Ahorngneis \\
\hline & Quelle am Grierbach (IV) & QGB & Hochstegenformation \\
\hline \multirow{7}{*}{$\begin{array}{l}\text { Weitere Quellen } \\
\text { des Tuxertals, } \\
\text { hydrochemisch } \\
\text { exemplarisch für } \\
\text { geologische } \\
\text { Einheiten }\end{array}$} & Brandalmquelle & BRA & Seidelwinklformation \\
\hline & Klausbodenquellen & KLA & Seidelwinklformation \\
\hline & Guggerquellen & GUG & Seidelwinklformation \\
\hline & Höllensteinquellen & HÖQ & Hochstegenformation \\
\hline & Zirbentalquelle & ZTQ & Hochstegenformation \\
\hline & Tuxbachquellen, Austritt Nr. 5 & TQ5 & Hochstegenformation \\
\hline & Grinbergquelle (Hangschuttquelle Grinbergalm) & GBQ & Ahorngneis (Hangschutt) \\
\hline
\end{tabular}

sungen und überwiegend konventionelle hydrogeologische Methoden eingesetzt. Neben den physikochemischen Messungen und hydrochemischen Analysen zur Charakterisierung werden andere Quellen des Gebietes untersucht, um die Hydrogeochemie der möglichen Aquifere zu typisieren (Tab. 2).

\section{Arbeitsgebiet}

\section{Geologie}

Das Arbeitsgebiet liegt am Nordwestrand des Tauernfensters (Abb. 1) und ist geprägt von einer NW einfallenden tektonischen Deckenstruktur. Den Kern des Tuxer Hauptkamms bildet der Venediger Deckenkomplex (Schmid et al. 2013) mit den nach ihrer Antiklinalform genannten ,Gneiskernen“ und ihren Hüllgesteinen (Lammerer et al. 2008). Ins Hangende folgen nach NW die Wolfendorndecke und der Glocknerdeckenkomplex (Frisch 1974). Die Carbonatreichen Einheiten der steil nach NW einfallenden Decken sind teilweise verkarstet und bieten damit hydraulisch präferenziell andere Abflussrichtungen als die tieferliegenden Gneise mit hydraulischen Vorzugsrichtungen entlang E-WScherzonen.

Die Ausgangsgesteine der Gneiskerne sind überwiegend in paläozoische Kontinentalkrustengesteine intrudierte Granite und Granodiorite, die mitsamt den hangenden Sedimentiten anschließend in mehreren Phasen deformiert und metamorph überprägt wurden (Schmid et al. 2013). Nach der alpidischen Subduktion, Kollision und Faltung wurde das Tauernfenster durch Wirken mehrerer Mechanismen im Miozän exhumiert (Rosenberg und Berger 2009; Neubauer et al. 1999; Schneider 2014), insbesondere auch mittels Deckenabschiebung und starker Erosion (Selverstone 1988; Rosenberg und Garcia 2011, 2012; Fügenschuh et al. 2012).

Die Gneiskerne - hier Ahornkern und Tuxer Kern (Abb. 1a) - sind E-W ausgelängte stehende Antiformen deren Schieferung im Eozän durch die frühalpidische Foliation stark geprägt wurde (Finger et al. 1993; Kurz et al. 2000; Schneider et al. 2013). Eine zweite oligozäne-miozäne Foliation (Barnes et al. 2004; Glodny et al. 2008) erzeugte die subvertikalen Achsenflächen und die Trennung der Kerne durch sinestrale Scherzonen (Schneider 2014; Rosenberg und Schneider 2008; Töchterle et al. 2011). Schneider et al. (2013) fassen die Ahornscherzone im Arbeitsgebiet und die Olperer Störungszone (Abb. 1a) am Rand zusammen mit vielen kleineren Scherzonen in den Gneiskernen als Tuxer Scherzonen in einem System zusammen. Dieses System ermöglicht Zirkulation von Grundwasser präferenziell in Richtung der Schieferungsund Störungsebenen in den ansonsten kaum durchlässigen Gneiskernen (Heldmann et al. 2019).

Der auf den Gneiskernen hangende Arkosegneis der Wustkogelformation (Abb. 1a) entstand aus permo-triassischen klastischen und vulkanoklastischen Sedimenten (Schön und Lammerer 1990). Er ist überwiegend aus Plagioklas, Chlorit und variablen Gehalten an Phengit, Quarz 
und Pyrit zusammengesetzt (Ledoux 1984). Die Paragneise der Wustkogelformation sind auch Teil anderer Decken wie der Modereckdecke bestehend aus Seidlwinklformation und Wustkogelformation, eingefaltet an der Basis der Bündnerschiefer des Glocknerdeckensystems (Brandner et al. 2008) (Abb. 1a). Gneise triassischen Alters finden sich außerdem in der Porphyrmaterialschieferserie der Wolfendorndecke (Frisch 1974; Beil 1986), die sich chemisch ähneln und sich vergleichbar auf die Hydrochemie der Grundwässer auswirken.

Die jurassische Hochstegenformation (Thiele 1970; Kiessling 1992), hauptsächlich Kalk- und Dolomitmarmore, lokal mit einem Quarzitband an der Basis (Frisch 1974), liegt in einigen Bereichen konkordant auf den Gneiskernen (sogenannte „Hochstegenzone“). Durch Isoklinalfaltung während der alpidischen Subduktion (Schmid et al. 2013), Faltung durch die sinistralen Scherzonen (Töchterle et al. 2011) und deckeninterne Überschiebungen variiert die Mächtigkeit zwischen wenigen zehner und mehreren hundert Metern. Da sich die Hüllen der beiden Kerne überlagern, und die Formation in der Wolfendorndecke ebenfalls vorkommt, ist die Formation nördlich des Grinbergs dreifach gestapelt mit insgesamt über vierhundert Metern Mächtigkeit, allerdings unterbrochen durch Phyllite und den Porphyrmaterialschiefer (Abb. 1a). Hydrogeologisch ergibt sich damit die Situation einer in Bezug zu ihrer Ausdehnung schmalen Wechsellagerung verkarstungsfähiger Gesteine zwischen geringleitenden Kluftgrundwasserleitern.

An der Basis des Glocknerdeckensystems sind verschiedene Metacarbonate triassischen Alters in die Deckenstapelung eingefaltet. Die Dolomite der Seidlwinkelformation (Schmid et al. 2013) bzw. zellig verwitterte Dolomite und Rauhwacken aus Flachwassercarbonaten der Aigerbachformation (Brandner et al. 2008; Lammerer et al. 2008), bilden neben dem Hochstegenmarmor weitere verkarstungsfähige Einheiten zwischen Finkenberg (in der Modereckdecke) bis zum Tuxer Joch (Schöberspitzenfaltung, Brandner et al. 2008) (Abb. 1a). Die Verteilung dieser Lagen ist allerdings durch starke Verfaltung irregulär und nicht durchgängig, wobei die hydraulische Durchlässigkeit nur in den eingefalteten Carbonaten nennenswert scheint.

Die darauf hangende, überwiegend aus klastischen Sedimenten stammende, kretazische Kasererformation der Wolfendorndecke besteht im Wesentlichen aus Schiefern und Phylliten (Thiele 1970; Frisch 1974). Diese sind ebenso wie die auflagernden Bündnerschiefer der Glocknerdecke mittel- bis engständig geklüftet, wobei auch die dort eingeschalteten Kalkphyllite nicht verkarstungsfähig sind.

Bereits Burger et al. (2007) kamen bezüglich der hydrogeologischen Erkundung für den Brenner Basistunnel am Westrand des Tauernfensters (Abb. 1a) zu dem Schluss, dass unter den Gesteinen des Arbeitsgebietes der Hochste- genmarmor der Hochstegenzone, die Triascarbonate (Seidlwinklformation) der Modereckdecke sowie die Störungen der Tuxer Scherzonen im Zentralgneis - im Fall der Olperer Störzone allerdings nur eingeschränkt - die einzig wesentlichen Grundwasserleiter bilden. Damit bilden die grundwasserstauenden Schiefer und die verkarsteten Carbonate unter hydraulischen Aspekten eine kontrastreiche Wechsellagerung.

Abweichend zur kompilierten geologischen Karte (GBA 2011) ergaben eigene Geländebefunde, dass die Phyllitlamelle (ca. $1,5 \mathrm{~km} \mathrm{~N}$ der Grinbergspitze bis zu $30 \mathrm{~m}$ mächtig) zwischen Metacarbonaten des Ahornkerns und Tuxer Kerns weder am Schmittenberg, noch im Bereich Finkenberg (Sass et al. 2016a; Sass und Schäffer 2018) die Carbonate trennt. Insofern wird angenommen, dass die Phyllitschuppe in der Tiefe gänzlich ausstreicht und die potenziellen Aquifere hydraulisch nicht getrennt sind.

\section{Hydrologie des Einzugsgebiets}

Der Niederschlag beträgt im Tal in Hintertux $1090 \mathrm{~mm} \mathrm{a}^{-1}$ (gemessen seit 2013, Messtation Nr. 14912 der Zentralanstalt für Meteorologie und Geodynamik ZAMG) und am Tuxer Hauptkamm 1350-1550 $\mathrm{mm} \mathrm{a}^{-1}$ (Bucher et al. 2004). Die potenzielle Evapotranspirationsrate beläuft sich für dieses hochalpine Tal- bis Bergklima etwa auf $270-330 \mathrm{~mm} \mathrm{a}^{-1}$ (Fecht et al. 2009). Das Gebiet mit steilen Hängen und bergauf zunehmend geringer Vegetation und Bodenbedeckung hat ein sehr geringes Wasserrückhaltepotenzial, weshalb der Anteil des Direktabflusses nach Regenfällen $80 \%$ übersteigen kann (Markart et al. 2011, 2015).

Die Entwicklung der Gletscher ist ein wichtiger Faktor der Hydrologie im Tuxertal, da die Gletscher der Zillertaler Alpen von 1969 bis 2011 bereits 61,8\% ihrer Gesamtfläche verloren hatten und der Rückgang wie überall in den Alpen fortschreitet (Paul et al. 2011, Fischer et al. 2015). Entlang des Tuxer Hauptkammes nehmen Anzahl und Fläche der Gletscher und Blockgletscher ausgehend vom Olperer Richtung Grinbergspitze stark ab, und NE der Realspitze liegen fast ausschließlich Blockgletscher vor. Gletscher verzögern den Abfluss, vor allem der Winterniederschläge, erhöhen die Speicherverluste durch ihr Schrumpfen und beeinflussen mit diesen (jahreszeitlichen) Kurzzeiteffekten nach Jansson et al. (2003) vermutlich auch im Arbeitsgebiet die Grundwasserneubildung. Gleiches gilt entsprechend für Blockgletscher (Krainer et al. 2007); insbesondere tragen die lokalen Gletscher mit erheblichem Anteil zur Grundwasserneubildung in den kristallinen Kluftwasserleitern am Tuxer Hauptkamm bei (Heldmann et al. 2019, Schäffer et al. 2020).

Es ist hinsichtlich des Verkarstungspotenzials auffällig, dass sämtliche Einzugsgebiete der Seitentäler südlich des Tuxbachs ihren Anfang auf den steilen kristallinen Gestei- 
nen nehmen und die Bäche somit niedrig mineralisiertes Wasser nach NW über die Metacarbonate der hangenden Decken führen.

\section{Methoden}

Zur Untersuchung von Grundwasser im alpinen Bereich gibt es klassische hydrogeologische Methoden, die vor allem natürliche Tracer beinhalten (Hilberg 2016; Kilchmann et al. 2004). Diese wurden verwendet, um die Herkunft der Thermalquellen im Kontext ihrer klimatischen und geologischen Rahmenbedingungen zu interpretieren. Zur weiteren Beurteilung wurden Geoindikatoren herangezogen, wie sie für die geothermische Prospektion üblich sind (Powell und Cumming 2010). Stabile Wasserisotope dienen der Eingrenzung des Einzugsgebietes. Geothermisch-geochemische Indikatoren und Mineralsättigungsindices werden eingesetzt, um die Hypothesen anhand der hydrogeochemischen Aquifereigenschaften zu verifizieren.

Die Quellen und die Grundwasseraustritte in den Tunneln wurden ausgewählt, um den Einfluss derjenigen Grundwasserkörper auf das Grundwasser zu untersuchen, die für die Genese der Thermalquellen wahrscheinlich ursächlich sind. Einige Quellen wurden aus räumlichen Gegebenheiten einzelnen Hypothesen zugewiesen (Tab. 2).

\section{Datenquellen}

Es gibt aktuell zwei mehrjährige Zeitreihen über Schüttung und Temperatur von einigen genutzten Thermalquellen, die von den Eigentümern zur Verfügung gestellt und im Rahmen dieser Studie genutzt wurden. Beide Messreihen wurden zur Beweissicherung begleitend $\mathrm{zu}$ Wassertunnelprojekten durchgeführt, die Messreihe 1969-1974 während dem Bau der Oberen Tuxbachüberleitung (OTU, Abb. 1b) und die andere 2014-2019 begleitend zur Unteren Tuxbachüberleitung (UTU, Abb. 1b). Zudem wurden Einzelmessungen 1994-2011 der jährlichen Trinkwasseruntersuchungen genutzt. Insgesamt liegen damit vor allem für die schüttungsstärksten und wärmsten Quellen Schüttungs- und Temperaturdaten für 332 Messtage sowie 89 hydrochemische Analysen vor.

\section{Eigene Messungen und Analysen}

Hydrochemische Feldparameter wurden seit 2011 mehrmals im Jahr an Thermalquellen und anderen Quellen des Tuxertals für diese Studie untersucht. Gemessen wurden Schüttung (Stoppuhr, Gefäß), Temperatur, ELF, pH, Eh und Sauerstoffsättigung (HQ40D-Multi, HACH). Hydrogencarbonat wurde vor Ort mit einem Digitaltitrator $(\mathrm{HACH})$ titriert. Die zugehörigen Wasserproben wurden am Institut für Geowissenschaften in Darmstadt auf typische Kationen $\left(\mathrm{Li}^{+}, \mathrm{Na}^{+}, \mathrm{NH}_{4}^{+}, \mathrm{K}^{+}, \mathrm{Mg}^{2+}, \mathrm{Ca}^{2+}, \mathrm{Sr}^{2+}\right)$ und Anionen $\left(\mathrm{F}^{-}\right.$, $\left.\mathrm{Cl}^{-}, \mathrm{Br}^{-}, \mathrm{NO}_{2}{ }^{-}, \mathrm{NO}_{3}{ }^{-}, \mathrm{PO}_{4}{ }^{2-}, \mathrm{SO}_{4}{ }^{2-}\right)$ mit Ionenaustauschchromatographie (IC) (HPIC 882 Compact IC Plus, Metrom), auf Fe und Mn mit Atomabsorptionsspektrometrie (AAS) (ContrAA 300, Analytic Jena) und auf Silizium mit Fluoreszenzspektroskopie analysiert. Diese Wasserproben wurden dazu in je zwei getrennten Polyethylenflaschen gekühlt transportiert, wobei die Proben zur Untersuchung der Kationen mit Salzsäure bis $\mathrm{pH}<2$ stabilisiert wurden. Die aufgezählten Methoden zählen zu den Standardwerkzeugen der Hydrogeologie (z. B. Langguth und Voigt 2013; Wisotzky et al. 2018).

\section{Geothermisches Potenzial}

Eine Berechnung des geothermischen Potenzials für den Aquifer wie für eine Tiefengeothermische Untersuchung üblich (VBI 2013) kann ohne 3D-Modell für den Untergrund, insbesondere den Hochstegenmarmor, und ohne genaue Temperatur-Tiefen-Verteilung nicht erfolgen. Zudem wäre ein solcher Ansatz zusammenhanglos für die Nutzung der natürlichen Quellen, insofern Erschließungsbohrungen keine aktuell erwarteten Maßnahmen sind. Als Ansatz wurde für ein Wärmetauscherszenario die potenziell verfügbare Energie errechnet. Auf Basis der Schüttungsraten aller Austritte und deren Temperaturen wurden mittels Annahmen zu den maßgeblichen Rahmenbedingungen - Nutztemperatur-, Abkühlungstemperaturniveau und daran angepasste Jahresarbeitszahlen aktueller Wärmepumpen - jährliche Entzugsmengen errechnet.

\section{Geothermische Indikatoren und Mineralsättigung}

Die hydrochemische Zusammensetzung von nicht-juvenilen Grundwässern wird von den thermo-chemischen Stabilitätsbedingungen im Aquifer bestimmt. Für hydrothermale Systeme sind je nach Mineralogie und involvierten Kationenaustauschreaktionen unterschiedliche Geothermometer sinnvoll (D’Amore und Arnórsson 2000). Zwei ternäre Diagramme wurden zur Interpretation verwendet, wobei das erste (TCSH nach Giggenbach und Goguel 1988; Giggenbach 1991) mit der Darstellung von Cl- $\mathrm{SO}_{4}-\mathrm{HCO}_{3}$ unter Berücksichtigung geringer Fluoridkonzentrationen identisch mit dem Kationenbereich des Piperdiagramms ist. Es gibt typische Bereiche im TCSH-Diagramm für Fluide spezifischer geothermaler Systeme, wobei reine vulkanische Dampfsysteme und reine Carbonatgesteine nicht abgebildet werden können (Powell und Cumming 2010). Im TNKMDiagramm (Giggenbach 1991) wird ein modifiziertes $\mathrm{Na}$ $\mathrm{K}-\mathrm{Mg}$-Verhältnis dargestellt, wie es aus dem Natrium-Kalium- und Natrium-Magnesium-Geothermometern für silikatische Reservoire abzuleiten ist. 
In zwei weiteren Diagrammen werden temperaturabhängige Mineralsättigungsindices verwendet, die mit PhreeqC auf Basis der thermodynamischen Datenbank von PhreeqC berechnet wurden (Parkhurst und Appelo 2013). Das SI-Calcit-pCO $\mathrm{p}_{2}$-Diagramm (Hilberg 2016) gibt anhand der Gleichgewichtsbedingungen von Carbonat und $\mathrm{CO}_{2} \mathrm{Hin}-$ weise zur Ähnlichkeit von Wässern hinsichtlich der Vegetation der Einzugsgebiete - entsprechend der Höhenlage - sowie der Carbonatverfügbarkeit in den durchflossenen Aquiferen. Die Siliziumkonzentration ist in der Regel direkt temperaturabhängig (Verma 2000), da durch die dominante Verbreitung von silikatischen Mineralen die Gleichgewichtsreaktion in großen Einzugsgebieten nahezu unabhängig vom weiteren Mineralbestand ist. Insofern wird über die Temperatur-Konzentrations-Kurve ersichtlich, ob die Wassertemperatur am Austritt der Temperatur im Reservoir entspricht, oder das Wasser thermisch equilibriert ist, was auf kurze Verweilzeiten oder Mischungen verschiedener Herkunft hindeuten kann.

\section{Stabile Isotopen}

Für die Herkunft der Thermalwässer können drei mögliche Quellen in Betracht gezogen werden, die unterschiedliche
Auswirkungen auf die Isotopenverhältnisse $\delta^{18} \mathrm{O}$ und $\delta D$ haben: Erstens Niederschläge und Versickerungen der nahen und weiteren Umgebung, zweitens Schmelzwässer der Gletscher, drittens Beiträge durch (alte) tiefe Formationswässer (Moser et al. 1980).

Die Zusammensetzung der stabilen Isotope im Niederschlag wird in dieser Region durch den saisonalen und den Höheneffekt beeinflusst. In diesem Gebiet ist der Einfluss des saisonalen Effekts wesentlich stärker. Außerdem tragen die Winterniederschläge, die nur etwa $40 \%$ der Jahresgesamtniederschläge ausmachen, mit ca. $60 \%$ an der Grundwasserneubildung unverhältnismäßig höher bei, als das Verhältnis zu den Sommerniederschlägen vermuten lassen würde (nur ca. 35-45\% gesamt) (Heldmann et al. 2019). AuBerdem ist der mögliche Einfluss von Blockgletschern und Gletschern auf das Isotopenverhältnis des Abflusses (Krainer et al. 2007) und durch deren Abflussverzögerungswirkung bei Niederschlägen (Winkler et al. 2016) für das in diesem Fall zunächst unbestimmte Einzugsgebiet naturgemäß nicht zu beziffern. Dementsprechend ist ein direkter Rückschluss auf die mittlere Höhe des Einzugsgebietes in diesem Fall nicht möglich.

Die Darstellung benachbarter Quellen ermöglicht allerdings trotzdem eine zweckmäßige relative Einordnung. $\mathrm{Zu}-$

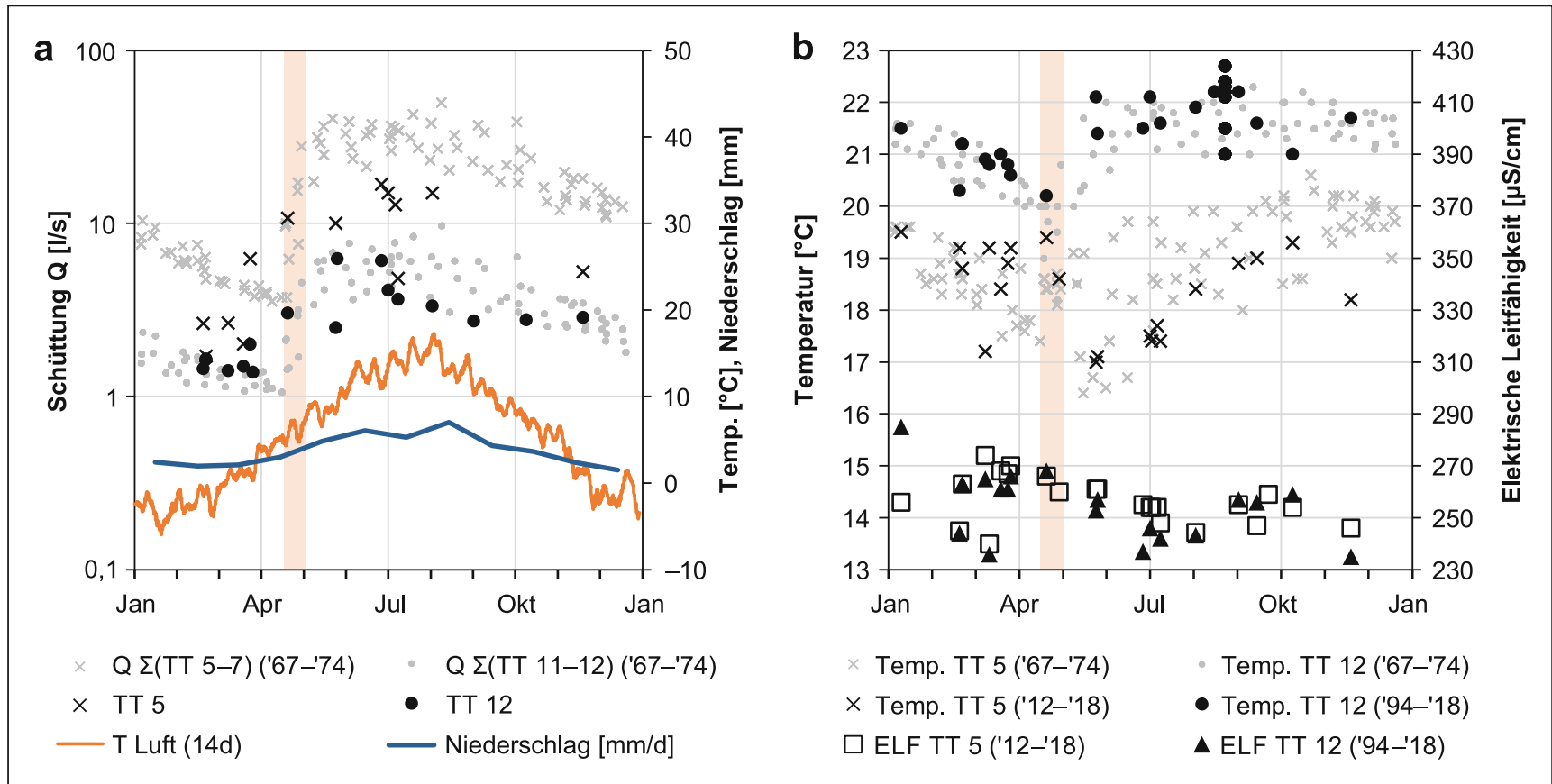

Abb. 2 Kennwerte ausgewählter Thermalquellen (TT5 und TT12) im Jahresgang mit Daten verschiedener Messreihen, mit dem hervorgehobenen Zeitraum des Schüttungsanstiegs Ende April (rötlich). a Schüttungsraten (Q) 2011-2018 im Vergleich zu verfügbaren historischen Schüttungssummen verschiedener Quellen (1967-1974), sowie durchschnittliche tägliche Lufttemperaturen und Monatsmittel der Tagesniederschläge in Hintertux (2014-2019, Daten von ZAMG). b Wassertemperatur (Temp.) und elektrische Leitfähigkeit (ELF) der Quellen

Fig. 2 Seasonal parameters of selected thermal springs (TT5 and TT12) collected from several data series, with period of discharge increase in April highlighted (red). a Discharge (Q) 2011-2018 and available historical sums of discharge for springgroups including TT5 and TT12 with daily air temperature and monthly average of daily precipitation in Hintertux (2014-2019, data provided by ZAMG). b Water temperature (Temp.) and electric conductivity (ELF) 
dem lassen die Darstellung der Wässer zusammen mit der lokalen Isotopenlinie (Heldmann et al. 2019) Rückschlüsse auf Fraktionierungen (Clark und Fritz 2013) durch Einflüsse nicht-meteorischer Wässer oder mögliche Mineralreaktionen zu. Die Proben der Quellwässer wurden mit Cavityring-down-Spektroskopie (CRDS) in einem L 2130i (Picarro) analysiert. Zum Vergleich wurden auch Durchschnittswerte der Langzeit-Isotopenverhältnisse der nächstgelegenen Messstationen Patscherkofel (IAEA und WMO 2018) und Gries am Brenner (Kralik et al. 2003; ANIP 2015) dargestellt.

Die Schwefelisotope der Formationen im Liegenden der Thermalquellen wurden an Mineralen und Wässern untersucht, um die Herkunft des im Thermalwasser vorhandenen Sulfats zuzuordnen. Da Pyritverwitterung eine häufige Sulfatquelle in Nichtevaporiten ist, wurden $\mathrm{zu}$ diesem Zweck Pyrit aus der Hochstegenformation und der Wustkogelformation (im Tunnel Obere Tuxbachüberleitung OTU) gesammelt und je zwei Mischproben analysiert. Da der Ahorngneis geringere Pyritgehalte hat, wurde für diese Formation ein Wasseraustritt im Tunnel Obere Tuxbachüberleitung (OTU) beprobt. Das Sulfat der Wasserproben wurde mit Barium gefällt und wie der Pyrit am Isotopenlabor der Universität Tübingen aus der Festphase analysiert. Die Genauigkeit beträgt $\pm 0,3 \delta^{34} S$ und $\pm 5 \%$ für den Schwefelgehalt.

\section{Ergebnisse}

\section{Schüttung}

Nur an den Quellen TT5, TT7, TT11, TT12, TT13 können Schüttungsmessungen mit 10\% Genauigkeit durchgeführt werden, denn nur vier Austritte sind überhaupt gefasst, die meisten anderen treten diffus aus und fließen verästelt über die Wiesen in den wenige Meter tiefer liegenden Tuxbach. Deshalb werden die an Stichtagen ermittelten Gesamtmengen mit einem größeren Streubereich angegeben, als die gezeigten Einzelquellen (Abb. 2). Die Schüttung der Thermalquellen (Abb. 2a) zeigt einen stark saisonal geprägten Verlauf mit einem sprunghaften Anstieg im April (Abb. 2a, rot hervorgehoben: 01.04.2015, 20.04.2016). Dieser Anstieg, der bei Quellen in hochalpinen Gebieten üblicherweise in Zusammenhang mit Schneeschmelzereignissen im Einzugsgebiet steht, entspricht einer Verzögerung von mehreren Wochen zum Schmelzen der Schneedecken im Februar/März am Austritt der Thermalquellen in Hintertux (bspw. Tauwetter ab Anfang Februar in 2017, Abb. 2b). Die starken Sommerniederschläge (Juni-August, Abb. 2a) führen zu mitunter mehrtägigen Spitzenabflussraten, die im Herbst geringer ausfallen und in der Regel spätestens im Oktober enden. Über die Frostperiode im Winter bis Frühjahr entwi- ckelt sich die Schüttung durch die Entleerung des Aquifers rezessiv. Die Dynamik der Schüttung in Reaktion auf einen Starkregen $\left(175 \mathrm{~mm} / \mathrm{m}^{2}, 27 .-29.10 .2018\right)$ und einer vorangegangenen mehrwöchigen Trockenphase im Sommer 2018 (Abb. 2a) zeigt, dass die Quellen ausgesprochen sensibel auf ausbleibenden Niederschlag reagieren und Niederschlag bereits innerhalb von $24 \mathrm{~h}$ zu sprunghaften Anstiegen von mehr als 301/s führen kann.

Die summierte Gesamtschüttung aller Thermalquellen zeigt bei allen drei Stichtagsmessungen ähnliche Verhältnisse zu den Schüttungen der Referenzquellen K3 und TT12. Demzufolge nehmen Niederschläge neben dem stark saisonal geprägten Verlauf fast gleichermaßen Einfluss auf alle warmen Quellen in Hintertux.

\section{Physikochemische Eigenschaften}

Die Temperaturen der Quellen zeigen saisonal geprägte Verläufe (Abb. 2) mit kurzfristigen Änderungen in Reaktion auf Niederschlagsereignisse. In der Frostperiode über den Winter hinweg zum Frühjahr nähern sich die Temperaturen einem konstanten Niveau von etwa $19-20^{\circ} \mathrm{C}$ an. Dabei gibt es zwischen der schüttungsstarken Quelle TT5 und der wärmsten Quelle TT12 einen auffallenden Unterschied: Sowohl der Aprilanstieg als auch kurzfristige Niederschlagsereignisse führen bei TT12 (TT11 u.a.) zu Temperaturanstiegen (bis $22,8^{\circ} \mathrm{C}$ ), aber bei TT5 (TT6 u.a.) zu sinkenden Temperaturen (bis $15,5^{\circ} \mathrm{C}$ ).

Die Änderungen der elektrischen Leitfähigkeit (Abb. 2b) zeigen hingegen in der Regel für alle Quellen gemeinsame Trends. Die elektrischen Leitfähigkeiten sind saisonal geprägt mit Maxima von bis zu $273 \mu \mathrm{S} / \mathrm{cm}$ im Februar/März und Minima von 235-245 $\mu \mathrm{S} / \mathrm{cm}$ im Juli/August. Die Effekte von Starkregenereignissen sind gering; so lässt sich nach dem Ereignis vom 27.-29.10.2018 $\left(175 \mathrm{~mm} / \mathrm{m}^{2}\right)$ nur eine Minderung der elektrischen Leitfähigkeit von ca. $10 \mu \mathrm{S} / \mathrm{cm}$ nach einer (TT12) bzw. zwei (TT5) Wochen später feststellen; ähnlich dem Ereignis Ende Juni/Anfang Juli 2017.

Mit pH-Werten von 7,9-8,3 sind die Thermalwässer schwach basisch. Die Sauerstoffsättigung liegt bei allen warmen Austritten meist über 90\%, sowohl bei gefassten als auch bei ungefassten Austritten. Zum Vergleich beträgt die Sauerstoffsättigung bei den kalten Austritten TKQ inmitten der Thermalquellen allerdings nur in einem Bereich von $40-60 \%$.

\section{Geothermisches Potenzial}

Bezüglich einer energetischen Nutzung der vorhandenen Quellen sind inklusive der bisher ungenutzten Austritte jahresdurchschnittliche Schüttungen von ca. 17-261/s (saisonales Minimum 5-81/s im Winter-Frühjahr) und jahresdurchschnittliche spezifische Temperaturen von $14-17^{\circ} \mathrm{C}$ 
Abb. 3 Piperdiagramm der Hauptionen für alle Lokationen Fig. 3 Piper plot of major ion composition

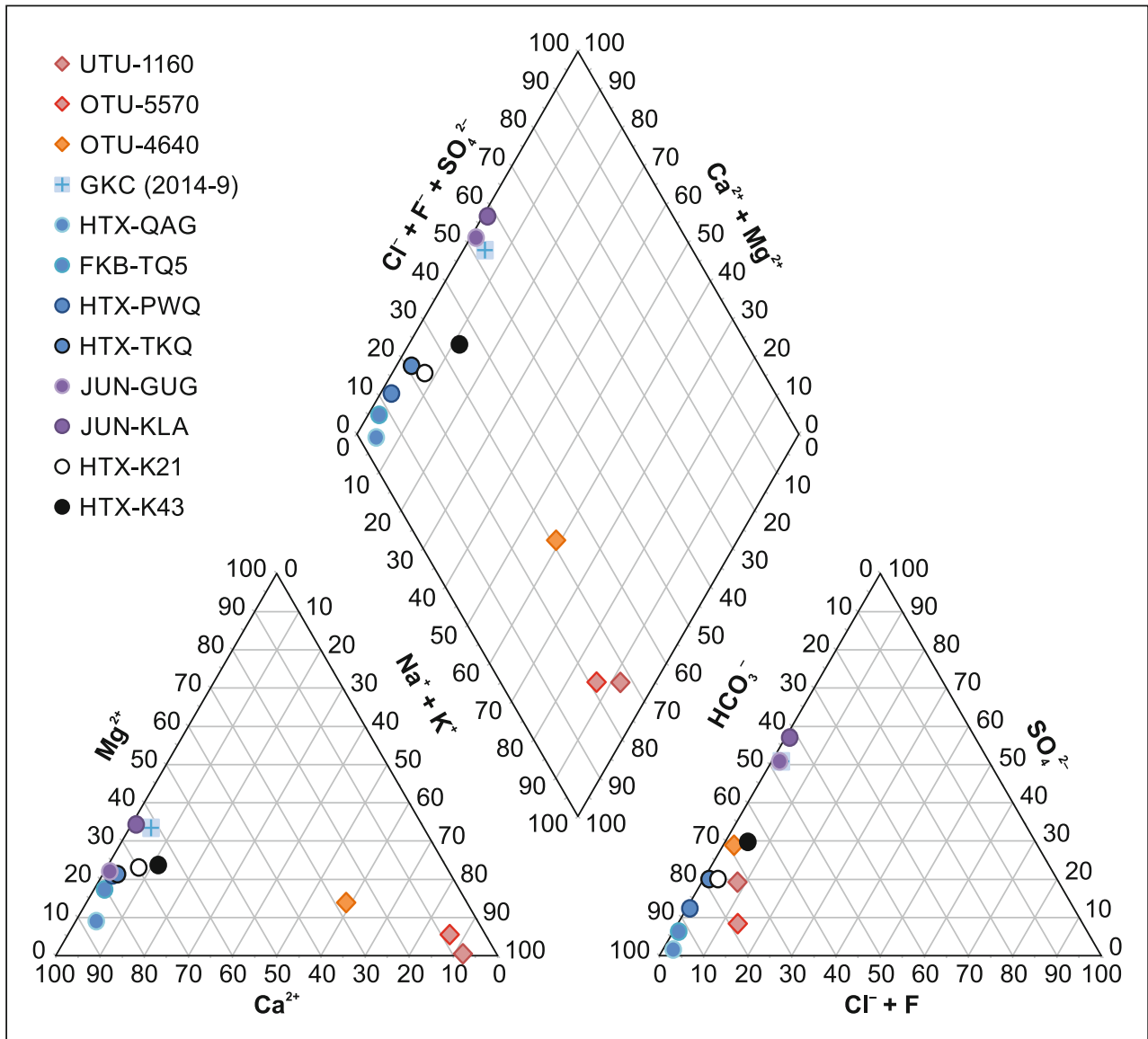

festgestellt worden. Je nach Temperaturniveau der Nutzung und Abkühlungsniveau des Thermalwassers sind unterschiedliche Wirkungsgrade und Jahresarbeitszahlen (JAZ) anzunehmen. Beispielsweise sind für balneologische Nutzungen $27-30{ }^{\circ} \mathrm{C}$ ausreichend, wobei für eine Abkühlung auf $4^{\circ} \mathrm{C}$ eine JAZ von 7 (VBI 2012) realistisch ist. Hingegen bei einer Nutztemperatur für Heizzwecke von $55^{\circ} \mathrm{C}$ wäre die $\mathrm{JAZ}$ etwa 5 , was einem aufzuwendenden elektrischen Anteil von $20 \%$ der verfügbaren Endenergie entspricht. In diesem Fall könnten mit oben genannten Größen der geothermischen Ressource jährlich ca. 1,0 GWh Heizenergie mittels Wärmepumpen bereitgestellt werden.

\section{Hydrochemie}

Die Stoffkonzentrationen in den Thermalquellen sind über den gesamten Untersuchungszeitraum ab 2011 ähnlich konstant wie die elektrische Leitfähigkeit, weshalb im Folgenden Mittelwerte der Thermalquelle TT 12 repräsentativ für alle warmen, bzw. TT 20 für alle gemischt-warmen, und TKQ für die kalten Quellen der Quellgruppe dargestellt werden.

Die Thermalquellen und die Quellen aus der Hochstegenformation sind $\mathrm{Ca}-\mathrm{HCO}_{3}-\mathrm{Wässer}$ (Abb. 3) wie sie typisch für oberflächennahe Grundwässer sind. Sowohl im Bereich Hintertux (Quelle am Grierbach QGB, Quelle Pferdeweide PWQ, TKQ), als auch im Bereich Grinberg/ Finkenberg (Zirbentalquelle, Grinbergalmquelle, Tuxbachquelle) zeigt ein Trend für zunehmende Lösungsinhalte zunehmende $\mathrm{Mg} / \mathrm{Ca}$-Verhältnisse innerhalb der Hochstegenformation. Dabei grenzen sich die Thermalquellen trotzdem deutlich durch höhere $\mathrm{Na}$-, $\mathrm{Mg}$ - und $\mathrm{Cl}$-Verhältnisse ab. Die gemischt-warmen Quellen (TT 20) liegen möglicherweise auf einer Mischungsgeraden (Abb. 3) zwischen den warmen Thermalquellen (TT 12) und bspw. einem Wasser mit der Zusammensetzung der Quelle Pferdeweide (PWQ).

Die Quellen der Triascarbonate gehören zum Ca-Mg$\mathrm{HCO}_{3}-\mathrm{SO}_{4}$-Wassertyp (Abb. 3). Allerdings fällt in diesen Bereich auch die Zusammensetzung des Großen Kunerbachs, dessen Einzugsgebiet Hochstegenformation, Kasererformation und Tuxer Gneiskern beinhaltet. Die Grundwasserproben aus den Tunneln zeigen, dass die Wässer aus den Paragneisen der Wustkogelserie (OTU-4640) und der Ahorngneise (UTU-1160, OTU-5570) vom Na-HCO ${ }_{3}$-Typ sind, wobei die Ahorngneiswässer zusätzlich noch erhöhte Cl-Gehalte zeigen (Abb. 4). 
Abb. 4 Schoellerdiagramm der Thermalquellen und ausgewählter Quellen potenzieller Grundwasserleiter

Fig. 4 Schoeller plot for thermal springs and springs related to their possible associated aquifers

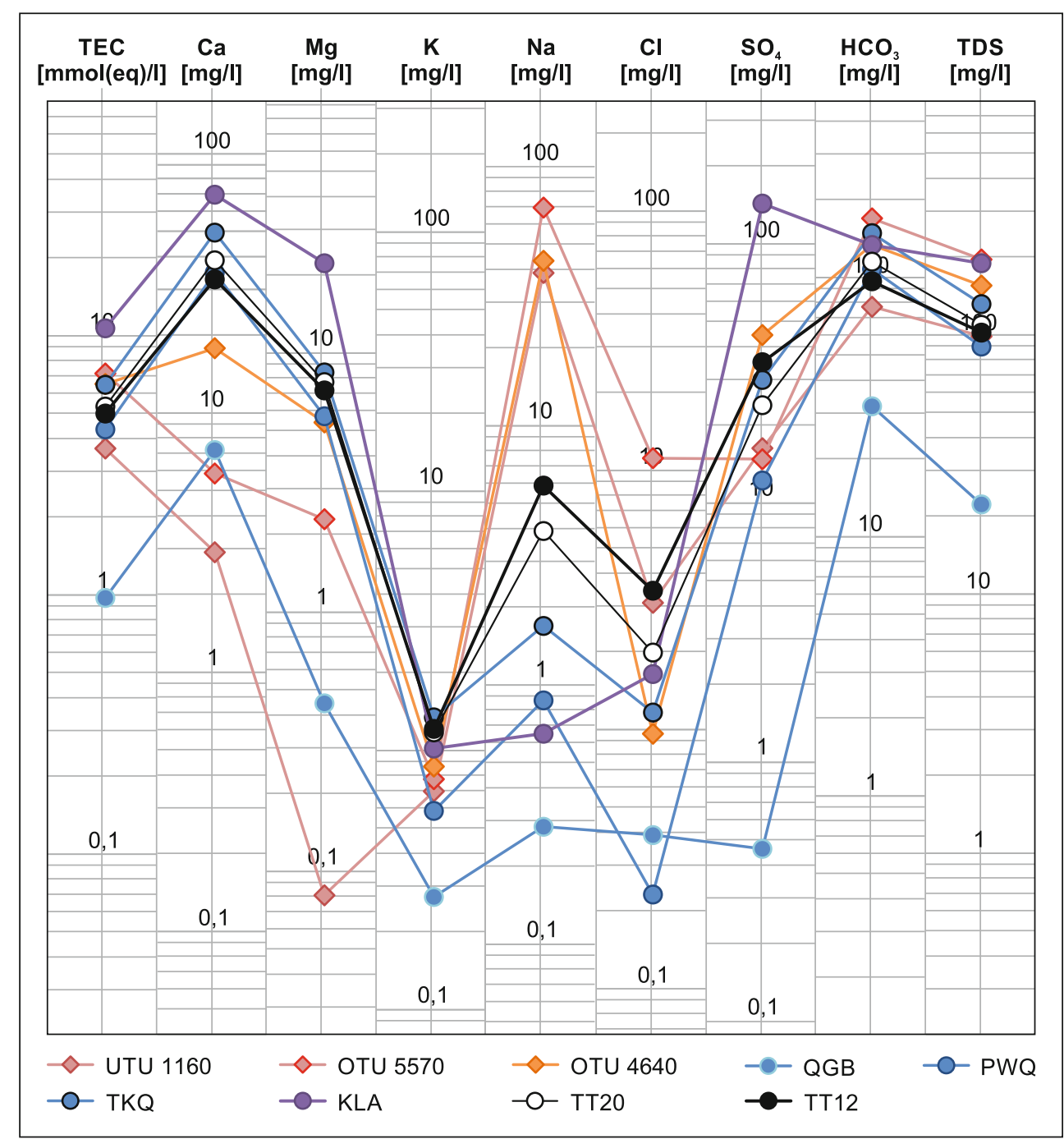

Die Darstellung im Schöllerdiagramm (Abb. 4) macht deutlich, dass die Thermalquellen verglichen mit anderen Quellen der Hochstegenformation mit ähnlicher oder höherer Leitfähigkeit (PWQ, TKQ) ähnliche $\mathrm{Ca}-\mathrm{Mg}$ - und $\mathrm{HCO}_{3}$.-Konzentrationen aufweisen. Allerdings sind die Naund Cl-Konzentrationen deutlich höher, etwa zwischen einem reiferen Ahorngneisgrundwasser (OTU 5570) und der Pferdeweidequelle (PWQ). Die Herkunft von $\mathrm{Na}$ und $\mathrm{Cl}$ ist nur in Wässern aus den Gneisen zu sehen. Einzige Alternative wären ein Mischungsanteil tiefer Grundwässer. Auch der Sulfatgehalt der Thermalquellen ist höher als bei allen Quellen des Hochstegenmarmors, und wird nur von den Wässern aus den Triascarbonaten (Seidlwinklformation) und der Wustkogelserie übertroffen.

\section{Geothermale Indikatoren und Mineralsättigung}

Eine Einordnung gemäß TCSH (Giggenbach und Goguel 1988) ergibt für die überwiegend $\mathrm{HCO}_{3}$-dominierten Wäs- ser keinen Hinweis auf Verbindungen zu einer möglichen Temperierung aus einem tieferen geothermischen Reservoir (Abb. 5). Eine Einordnung nach TKNM (Giggenbach 1991) klassifiziert alle untersuchten Wässer des Tuxertals als kalt bzw. geothermisch ungereift (,immature“) mit Ausnahme der etwas älteren Grundwässer aus dem Ahorngneis, die möglicherweise als partiell equilibriert eingestuft werden können, allerdings selbst nicht wärmer als die Thermalquellen sind.

Die Darstellung $\mathrm{CO}_{2}$-Partialdruck-Calcitsättigung ordnet die Thermalquellen in einen Bereich ein, der zugleich dem Bereich einer Gruppe tiefer Grundwässer aus dem Tunnel Obere Tuxbachüberleitung liegt, wohingegen benachbarte Quellen und andere Quellen des Hochstegenmarmors in andere Bereiche des Diagramms fallen (Abb. 6; Grenzen SI Calcit $\geq-1$ und $\mathrm{pCO}_{2} \leq-3$ nach Hilberg 2016). Die Grundwässer des Tunnels stammen aus Austritten auf etwa $1800 \mathrm{~m}$ ü. NHN, die nördlich des Tuxer Hauptkamms (Bereich mit Gipfel der Gefrorene Wand 3286m ü. NHN) tief 


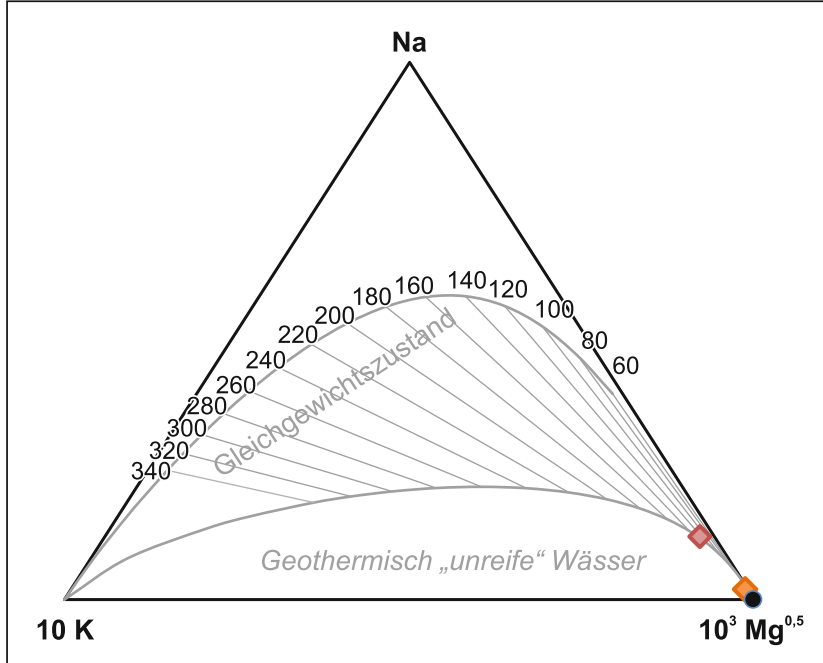

Abb. 5 TNKM-Diagramm von Wässern aus tieferen Grundwasserbereichen (alle außer UTU 1160 und OTU 4640 überlagen sich im MgBereich rechts unten)

Fig. 5 TNKM plot of springs from deep groundwater (all but UTU 1160 and OTU 4640 are sub-located in the lower right)

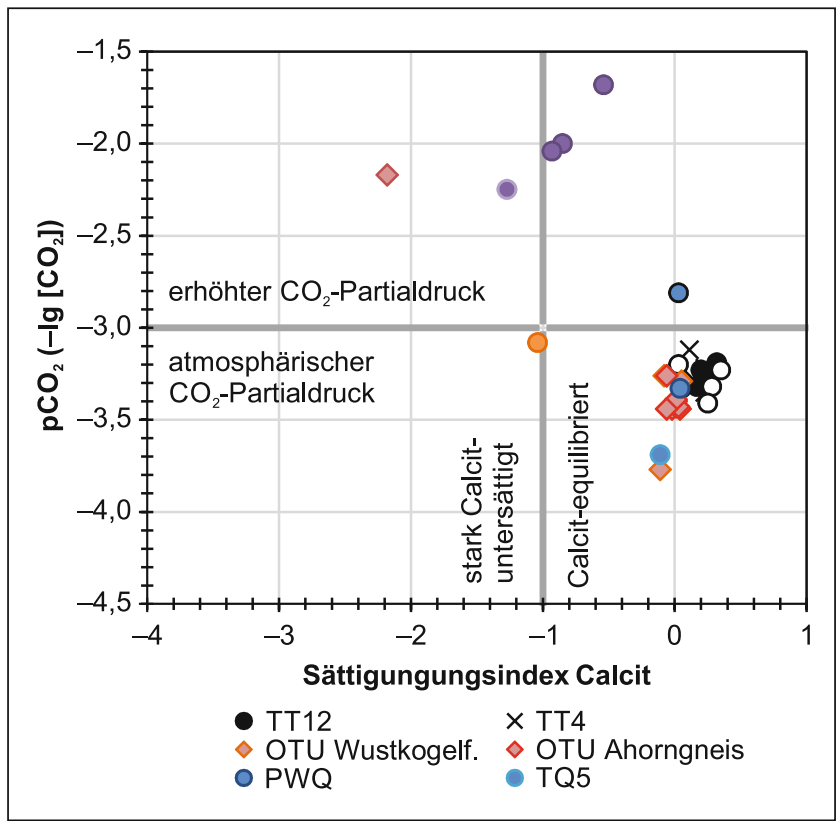

Abb. 6 Sättigungungsindex Calcit-pCO $\mathrm{pO}_{2}$-Diagramm nach Hilberg (2016)

Fig. 6 Saturation Index Calcite-pCO $\mathrm{pC}_{2}$ diagram after Hilberg (2016)

unterhalb der Interaktionsfläche Grundwasser-vadose Zone in den Zentralgneisen zirkuliert sind. Insofern ist der $\mathrm{CO}_{2}-$ Partialdruck Indikator für eine ähnliche, hochalpine Höhenlage des Einzugsgebietes, etwa entsprechend der Grierkarquelle. Hingegen der Thermalquellen haben andere Quellen, mit vergleichbaren Austrittshöhen nahe am Tuxbach, durch den Vegetationseinfluss aus ihren tieferen Einzugsgebieten deutlich höhere $\mathrm{CO}_{2}$-Partialdrücke. Die leichte Über-

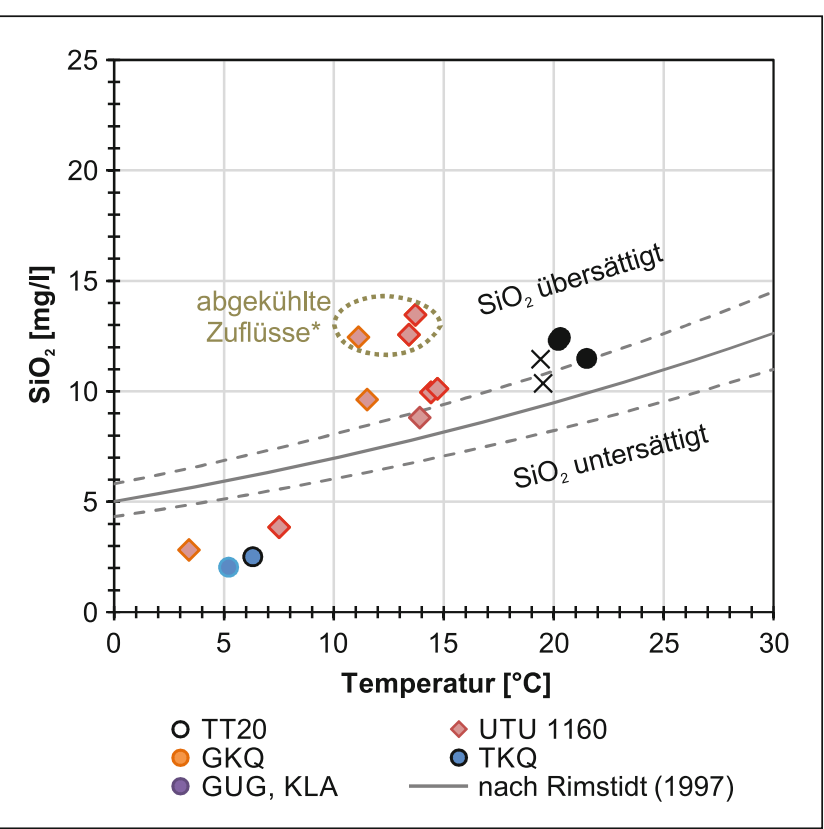

Abb. 7 Siliziumgehalt gegen Wassertemperatur von Wässern aus tieferen Grundwasserbereichen ( $\mathrm{SiO}_{2}$-Löslichkeit nach Rimstidt 1997) Fig. 7 Silica vs. surface temperatures of springs from deep groundwater

sättigung an Calcit ist plausibel mit einer Passage des häufig calcitischen Hochstegenmarmors in der Nähe des Quellaustritts erklärbar. Quellen aus der dolomitischen Seidlwinklformation (GUG, KLA) sind gegenüber den Thermalquellen stärker Calcit-untersättigt.

Die Tuxer Thermalquellen sind hinsichtlich ihres Siliziumgehaltes leicht übersättigt und vergleichbar mit den kristallinen Grundwässern, die allerdings etwas kühler sind (Abb. 7). Ein Teil der abgebildeten Quellen (Heldmann et al. 2019) entstammt Reservoiren mit mächtigerer Überdeckung (aus dem Bereich Hoher Riffler 1,5 km östlich des Tunnels) und wird von dort über Störungssysteme zum Tunnel geleitet und dabei abgekühlt, diese Wässer sind erkennbar übersättigt. Die Übersättigung ist ein weiterer Hinweis darauf, dass die Thermalwässer auf eine Mischung zweier bei unterschiedlichen Temperaturen equilibrierter Grundwässer zurückgehen können (je eine wärmer und eine kälter) oder zumindest abgekühlt wurden (von bspw. ca $25^{\circ} \mathrm{C}$ Reservoirtemperatur).

\section{Stabile Isotopen}

Die Tuxer Thermalquellen sind isotopisch leichter als die benachbarten Quellen (Quelle Pferdeweide PWS, Kaiserbründlquelle KBS) und die wärmsten Austritte sind mit $\delta^{18} \mathrm{O} 13,7 \%$ (Oktober) bis 14,0\%0 (Februar 2016) teilweise etwas leichter als die daneben austretenden kalten Quellen (PWQ, TKQ) (Abb. 8). Eine lokale Niederschlags-Wasserisotopenlinie (LMWL) aus einer Sammlung von Nie- 
Abb. 8 Wasserisotopenverhältnisse der Quellen von Hintertux zusammen mit gewichteten Jahresdurchschnittswerten der regionalen Niederschläge, gewichteten lokalen Sommerniederschlägen und Grundwässern aus dem Tunnel OTU. (*Daten nach Heldmann et al. 2019)

Fig. 8 Stable water isotopes of springs, groundwater seepage within the OUT tunnel, weighted mean precipitation from regional stations and from local summer precipitation in 2014. (*Data after Heldmann et al. 2019)

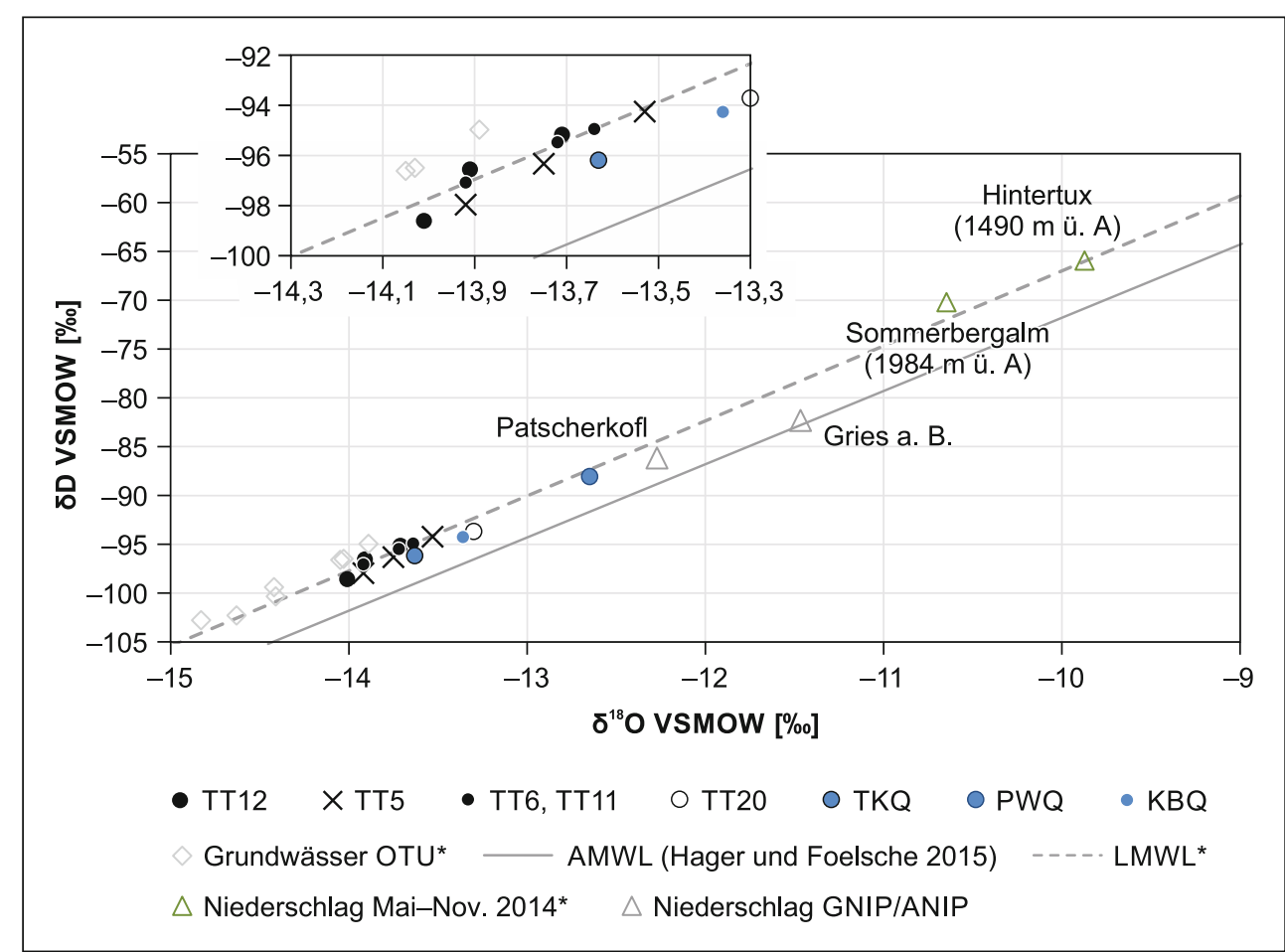

Tab. $3 \delta^{34} S$ aus Quellwässern und Gesteinen der Umgebung von Hintertux ( ${ }^{\text {adie }}$ Angaben zum Tunnel beziehen sich auf die Tunnelmetrierung (mfSr) ausgehend vom unteren Ausgang am Schlegeisspeicher in Richtung des Vortriebs NNW)

Table $3 \delta^{34} \mathrm{~S}$ from water samples of springs and mineral samples from rocks around Hintertux (arock samples from the Upper Tuxbach water tunnel are given with their distance from the southern tunnel exit in $\mathrm{m}(\mathrm{mfSr})$ in the direction of the tunneling progress)

\begin{tabular}{|c|c|c|c|c|}
\hline Probe & $\delta^{34} \mathrm{~S}[\% 0]$ & S-Gehalt $[\% \mathrm{~S}]$ & Analysematerial & Zugeordnete geol. Einheit \\
\hline Hintertuxer Thermalquellen & 6,63 & 13,0 & $\mathrm{BaSO}_{4}$ & - \\
\hline Pyrit aus Marmor Tuxbachquelle & $-26,09$ & 43,6 & $\mathrm{Fe} 2 \mathrm{~S}$ & Hochstegenfm \\
\hline Pyrit aus Marmor Tuxbachquelle & $-21,19$ & 45,1 & $\mathrm{Fe} 2 \mathrm{~S}$ & Hochstegenfm \\
\hline Pyrit im Tunnel OTU 4000 mfSr & $-4,17$ & 47,0 & $\mathrm{Fe} 2 \mathrm{~S}$ & Wustkogelfm \\
\hline Pyrit im Tunnel OTUa 6900 mfSr & $-2,41$ & 31,8 & $\mathrm{Fe} 2 \mathrm{~S}$ & Wustkogelfm \\
\hline Wasseraustritt in OTU $5160 \mathrm{mfSr}$ & $-2,68$ & 13,6 & $\mathrm{BaSO}_{4}$ & Ahorngneis \\
\hline
\end{tabular}

derschlagsproben des gleichen Arbeitsgebietes (Heldmann et al. 2019) zeigt einen starken Zusammenhang mit den lokalen Quellen. Es ist kein signifikanter Shift (Abweichungen sind kleiner als die Messabweichungen) von der Lokalen Niederschlagslinie festzustellen, weswegen weder Anteile aus fossilen Formationswässern noch eine Fraktionierung durch Mineralreaktion anzunehmen sind.

Alle gemessenen Quellwässer sind isotopisch leichter als die regionalen Niederschläge sowohl niedrigerer Messstationen (Gries am Brenner), als auch höherer (Patscherkofel) (Abb. 8). Insbesondere sind sie wesentlich leichter als die lokalen Niederschläge des Sommerhalbjahres (Mai-November) vermuten lassen, weshalb Beiträge isotopisch leichterer Herkunft, aus Winterniederschlägen oder Anteilen von Gletscherschmelzwasser, einen dominanten Anteil an der Grundwasserneubildung im Einzugsgebiet aller Hintertuxer Quellen haben müssen. Das trifft ebenso auf die im Tunnel Obere Tuxbachüberleitung (OTU) beprobten
Grundwässer (Heldmann et al. 2019) zu, wobei zu beachten ist, dass diese gegenüber den Quellen in Hintertux zu einem gewissen Teil durch den Höheneffekt beeinflusst sind, da sie etwa $300 \mathrm{~m}$ höher beprobt wurden.

Die Schwefelisotope der Thermalquelle weisen ein Isotopenverhältnis von $\delta^{34} \mathrm{~S}$ 6,63\% auf (Tab. 3). Der Schwefel aus den Pyriten der Hochstegenformation zeigt mit $\delta^{34} \mathrm{~S}$ $-26,1$ bis $-21,1 \%$ ein für marine Pyrite typisches, sehr leichtes Isotopenverhältnis. Die Pyrite der liegenden Paraund Orthogneise sind mit $\delta^{34} \mathrm{~S}-2,4$ bis $-4,2 \%$ in einem typischen Bereich für Kristallingesteine.

Der Schwefel in den Quellwässern könnte allerdings auch aus dem Sulfat triassischer Evaporite der Modereckdecke (Aigerbachformation, Brandner et al. 2008) oder auch aus strukturell substituiertem Sulfat in Carbonaten stammen, also aus Dolomit und Calcit der triassischen Seidlwinklformation oder der oberjurassischen Hochstegenformation. Der Sulfatgehalt in natürlichen Carbonaten 
beträgt etwa 0,02-2,4\% (Staudt und Schoonen 1995). Die Fraktionierung beim Einbau von Schwefel beträgt etwa $\delta^{34} \mathrm{~S}$ 0,7\%o gegenüber dem Meerwasser (Kampschulte und Strauss 2004). Die Isotopenverhältnisse in der Mittel- bis Obertrias waren denen im Oberjura zu ähnlich, jeweils $\delta^{34} \mathrm{~S} 13-20 \%$ (Kampschulte und Strauss 2004; Strauss 1997), um anhand der Quellwässer eine Unterscheidung der Ausgangsgesteine zu ermöglichen.

Demnach ist klar, dass das Sulfat im Thermalwasser nicht aus einem einzigen Mineral stammen kann. Je nach involvierter Geologie ergeben sich die Mischungsverhältnisse des Schwefels aus den Carbonaten gegenüber dem Schwefel aus den Pyriten anhand der Isotopenverhältnisse im Thermalwasser. Käme der gesamte Schwefel in den Thermalquellen aus der Hochstegenformation ergäbe (Hochstegencarbonat - Hochstegenpyrit) ein Carbonatanteil von $68-84 \%$. Bei einer Mischung von Wässern aus pyritfreier Hochstegenformation oder Modereckdecke mit Wässern aus den Gneisen (Carbonat/Sulfat - Ahorn-/ Wustkogelpyrit) ergibt sich ein Carbonatanteil 40-63\%. Damit ist der maximale Anteil an Sulfat aus den Gneisen im Thermalwasser 37-60\%, bzw. kleiner, weil eine zusätzliche Lösung von Pyrit im Hochstegenmarmor sehr wahrscheinlich ist.

\section{Diskussion}

Es wird deutlich, dass ein einzelner Zirkulationspfad nicht ausreicht, um gleichzeitig beobachtete Temperaturen, Schüttungsverhalten und Chemismus der Thermalquellen zu erklären. Die genaue Position der Störzonen bzw. der Übertritt des warmen Tiefenwassers aus den Gneisen in den Hochstegenmarmor zwischen Grieralm und NW-Hang Schmittenberg ist mit den verwendeten Methoden nicht genauer zu verorten. Im Wesentlichen liegt es also unter dem Schuttfächer des Schmittenlochs, der wegen anhaltender Steinschlagaktivität nicht zugänglich ist. Der Einsatz gängiger geophysikalischer Methoden der Karsterkundung (Chalikakis et al. 2011) steht damit vor dem Problem, dass der mögliche Einsatz durch sicherheitstechnische Aspekte eingeschränkt wird, außerdem neben dem Festgestein zusätzlich Lockergesteine des Schuttfächers durchdrungen werden müssen und zuletzt der Zutritt der Tiefenwässer unter den kalten Karstwässer maskiert sein kann. Andere Probleme ergeben sich für eine genauere Erkundung durch die steile Morphologie.

\section{Schlussfolgerungen}

Die kurzfristigen Reaktionen der Schüttungsraten sprechen für eine Verbindung der Thermalquellen zu einem Karst- system als wesentlichem Bestandteil ihrer Herkunft. Da das Tauen im Umfeld und an der oberhalb der Quellen gelegenen Nordwestflanke des Schmittenbergs keinerlei zeitgleichen positiven Effekt auf die Schüttungsraten zeigt, spielt dieses nahe Einzugsgebiet folglich kaum eine Rolle. Das ist auch dadurch belegt, dass selbst Starkregenereignisse, die zu einer Vervielfachung der Schüttung führen, keinen drastischen Abfall der elektrischen Leitfähigkeit mit sich bringen. Demnach muss das oberflächliche Einzugsgebiet in einiger Entfernung zu den Thermalquellen liegen, da der hydraulische Effekt zunächst unmittelbar das Wasser aus dem Aquifer-Reservoir drückt und die folgend zu beobachtende Verdünnung durch Vermischung stark gedämpft ist.

Eine mögliche Erklärung für die Zusammensetzung der Thermalwässer wird auf Basis hydrogeologisch regional typischer Grundwässer folgend gegeben: Ein Gneiswasser (aus Ahorn- oder Tuxer Gneiskern) mit einer Cl-Konzentration von beispielsweise $5 \mathrm{mg} / \mathrm{l}$ (zwischen UTU 1160 und OTU 5570) gemischt mit einem Wasser der Hochstegenformation, beispielsweise der Pferdeweidequelle, entspricht anhand der Hauptionen einem Mischungsverhältnis von ca. $52 \%$ zu $48 \%$ (mit Ausnahme des $\mathrm{Mg}$ ). Hätte das Carbonatwasser $6{ }^{\circ} \mathrm{C}$, müsste das Gneiswasser etwa $37^{\circ} \mathrm{C}$ haben, um die maximalen Temperaturen der Thermalquellen zu erreichen. Die genannte Temperatur des Gneiswassers benötigt bei einer Oberflächentemperatur von $0^{\circ} \mathrm{C}$ und einem alpinen geothermischen Gradienten von $0,022^{\circ} \mathrm{C} / \mathrm{m}$ (Vosteen et al. 2003; Heldmann et al. 2019) eine Überdeckung von 3180 mü. A. Durch das chemische Ungleichgewicht des Mischungswassers käme es zu überschüssigem $\mathrm{CO}_{2}$ und damit zu einer Mischungskorrosion im Hochstegenmarmor, wodurch $\mathrm{Ca}, \mathrm{Mg}$ und $\mathrm{HCO}_{3}$ bis zur Sättigung schneller gelöst würden. Wie die Zusammensetzung des Großen Kunerbachs (entsprechend dem Hochstegenmarmor im Einzugsgebiet) und Bohrungen in der unteren Hochstegenformation zeigen (Sass et al. 2016a), umfassen die Hochstegenmarmore durchaus auch größere Bereiche mit Dolomitmarmoren, aus denen $\mathrm{Mg}$ gelöst werden kann.

Anhand ihrer Hydrochemie und physikochemischen Parameter wird für die Herkunft der Tuxer Thermalquellen ein Zirkulationspfad hergeleitet und drei der vier oben genannten Hypothesen können demnach ausgeschlossen werden:

I. Ein Einzugsgebiet am Schmittenberg mit einer Temperaturerhöhung resultierend aus einem tiefen Zirkulationspfad durch den Hochstegenaquifer ist hydraulisch und geothermisch nicht plausibel. Gemäß einem lokalen geothermischen Gradienten im Hochstegenmarmor von $2,5^{\circ} \mathrm{C} / 100 \mathrm{~m}$ (Sass et al. 2016a) wäre ein Fließpfad in über $800 \mathrm{~m}$ Tiefe vonnöten. Die Carbonate der Hochstegenformation sind am Schmittenberg aber maximal 400m mächtig (Ledoux 1984). 
II. Die für die Fließwege im Untergrund zur Verfügung stehenden Tiefen im Hochstegenmarmor sind auch hier limitiert. Zudem müssten die Fließpfade sowohl den Tuxbach, als auch die Quelle auf der Pferdeweide unterqueren um bei den Thermalquellen auszutreten. Als Voraussetzung müsste der dort durchgängige, verkarstungsfähige Hochstegenmarmor ausgerechnet in diesem Bereich zwei Systeme hydraulisch abgrenzen; in der QPW wurden zu keiner Jahreszeit Temperaturen über $7^{\circ} \mathrm{C}$ gemessen. Damit ist auch diese Hypothese ohne Postulierung einer aus geothermischer (Wärmezufuhr aus tiefer liegenden Geokörpern) und chemischer (Na-Konzentration) Sicht unbedingt notwendigen weiteren Komponente nicht haltbar.

III. Eine Unterquerung des Tuxbachs ist als hydraulisches Modell ungewöhnlich aber denkbar, wenn der Keil der Kasererformation in der Tiefe ausstreicht. Der dadurch erzwungene tiefe Fließpfad von den Tuxer JochQuellen durch die Seidlwinkl-Carbonate könnte die benötigte Tiefenstufe beisteuern. Der Austritt durch die Hochstegenmarmore, könnte in diesem Fall durch die Geometrie der stauenden Kasererformation bedingt sein. Aber auch dieser Hypothese widerspricht der Natriumgehalt in den Thermalquellen. Auch die Existenz der kalten Quellen TKQ, die noch unterhalb der Thermalquellen austreten und eine für den calcitischen Hochstegenmarmor typische Zusammensetzung haben, widerspricht dieser Hypothese.

Die Möglichkeit aufsteigender Formationswässer aus tiefen Reservoiren als Ursache der Temperaturanomalie ist ebenso durch die H/O-Isotopenverhältnisse sowie geothermische Indikatoren auszuschließen. Damit bleiben ausschließlich nur Wässer aus den Gneisen des Ahornkerns um den Ursprung der Temperaturanomalie der Thermalquellen zu erklären.

Im Einklang mit allen Messungen und Beobachtungen erweist sich die Hypothese (IV) mit dem Einzugsgebiet im Grierkar, der Versickerung an der Grieralm und der Vermischung im Hochstegenmarmor unter dem NW-Hang des Schmittenberges mit Wässern aus den Tuxer Gneisen am wahrscheinlichsten. Möglich wäre auch ein noch weiterer Einzugsbereich Richtung Höllensteinalm. Der Transport im Hochstegenaquifer wird demnach in sehr ungewöhnlicher Weise entgegen der Fließrichtung des Tuxbachs erzwungen, durch die extreme hydraulische Anisotropie der Stockwerke und Einheiten der NE-SW-streichenden Decken. Die Temperaturspreizung nach Niederschlagsereignissen (im Grierkar) und der zeitlich versetzten Peaks der Verdünnung (bspw. in den Quellen TT5 und TT12) werden als Hinweis auf die vertikale Gliederung des Karstsystems gesehen. Dabei entspricht die Dynamik von TT5 den oberflächennäheren Grundwasserfließpfaden und TT12 den tieferliegenden.

Die Nutzung der natürlichen Thermalquellen als geothermische Ressource ist nur in begrenztem Rahmen erweiterbar und sinnvoll, da das jährliche Schüttungsminimum in die Hauptheizperiode fällt. Da aber die eigentliche Quelle der Temperaturanomalie eben nicht im Karstaquifer sondern darunter liegt, ist diese eigentlich nicht an dieselben jahreszeitlichen Schwankungen gebunden. Damit scheint das wirtschaftlich größere Potenzial in einer Brunnenbohrung, statt in einer neuen Fassung aller Quellen zu liegen. Oben genannte Annahmen für das tiefere Thermalwasser (Wassertemperatur $35^{\circ} \mathrm{C}$, Schüttungen $>101 / \mathrm{s}$ ) führen bei analogen Berechnungen zu Wärmepumpennutzung (JAZ 5) zu jährlich mehr als 1,4 GWh und das insbesondere weitgehend konstant auch über die Frostperiode.

Die verwendeten hydrogeochemischen Methoden machen eine geophysikalische Unterstützung für eine möglicherweise folgende Exploration zur weiteren Erschließung nicht obsolet. Aber durch diese hydrochemische Studie wurde durch die Präzisierung des Einzugsgebietes und Klärung der bisher unklaren Zirkulationspfade der zukünftige Aufwand deutlich reduziert. Ansatzpunkte und Ausrichtung für Bohrungen zur Fassung der Gesamtschüttung mit maximalen Temperaturen werden deshalb nicht hangwärts (SE) oder vertikal in die Tiefe unter dem Quellaustritt empfohlen, sondern entgegen der bisherigeren konzeptionellen Modelle, mehrere einhundert Meter Richtung NE bis in den unter der grundwasserstauenden Kasererformation liegenden Hochstegenmarmor. Als weitere Schritte werden zur Exploration eine Radonkartierung im Bereich des Schmittenbergs und zur Vulnerabilitätsanalyse ein Markierungsversuch der Grierkarschwinde empfohlen.

Danksagung Wir danken der Familie Kirchler, Eigentümer des Thermalbades in Hintertux, für die Bereitstellung von historischen Daten und den Zugang zu ihren Quellen. Die Schwefelisotope wurden vom Team des Isotopenlabors der Universität Tübingen analysiert. Weiterhin danken wir Frau Assoz.-Prof. Dr. Sylke Hilberg und einem unbekannten Reviewer für ihre konstruktive Kritik und hilfreiche Hinweise.

Funding Open Access funding provided by Projekt DEAL.

Open Access Dieser Artikel wird unter der Creative Commons Namensnennung 4.0 International Lizenz veröffentlicht, welche die Nutzung, Vervielfältigung, Bearbeitung, Verbreitung und Wiedergabe in jeglichem Medium und Format erlaubt, sofern Sie den/die ursprünglichen Autor(en) und die Quelle ordnungsgemäß nennen, einen Link zur Creative Commons Lizenz beifügen und angeben, ob Änderungen vorgenommen wurden.

Die in diesem Artikel enthaltenen Bilder und sonstiges Drittmaterial unterliegen ebenfalls der genannten Creative Commons Lizenz, sofern sich aus der Abbildungslegende nichts anderes ergibt. Sofern das betreffende Material nicht unter der genannten Creative Commons Lizenz steht und die betreffende Handlung nicht nach gesetzlichen Vorschriften erlaubt ist, ist für die oben aufgeführten Weiterverwendungen des Materials die Einwilligung des jeweiligen Rechteinhabers einzuholen. 
Weitere Details zur Lizenz entnehmen Sie bitte der Lizenzinformation auf http://creativecommons.org/licenses/by/4.0/deed.de.

\section{Literatur}

ANIP (Austrian Network of Isotopes in Precipitation): Österreichisches Netzwerk für Isotopen $(18 \mathrm{O}, 2 \mathrm{H}, 3 \mathrm{H})$ in Niederschlägen und Oberflächengewässern. Lebensministerium, Ämter d. Landesregierungen, AIT und Umweltbundesamt, Wien (2015)

Barnes, J.D., Selverstone, J., Sharp, Z.D.: Interactions between serpentinite devolatilization, metasomatism and strike-slip strain localization during deep-crustal shearing in the Eastern Alps. J. Metamorph. Geol. 22(4), 283-300 (2004)

Beil, F.: Petrographischer Bestand, Genese und Alter der „Porphyrmaterialschiefer" am Nordrand des Tauernfensters zwischen Hintertux und Gerlospass, Tirol. Doctoral dissertation. (1986)

Biemann, R.: Projekt der Wasserversorgungsanlage für das Hallenbad Hintertux, Josef Kirchler. - Unveröffentlichter Bericht (WB, Schwaz, Zl. 0574), 4 S., Jenbach (1969)

Brandner, R., Reiter, F., Töchterle, A.: Überblick zu den Ergebnissen der geologischen Vorerkundung für den Brenner-Basistunnel. Geo Alp 5, 165-174 (2008)

Bucher, K., Kerschner, H., Lumasegger, M., Mergili, M., Rastner, P.: Spatial precipitation modelling for the Tyrol region. Institut für Geographie, Univ, Innsbruck, S. 1-5 (2004)

Burger, U., San Nicolò, L., Bösel, D., Perello, P.: Hydrogeologische Modelle-Hilfsmittel für die Planung am Beispiel des Brenner Basistunnel. Computeranwendungen in Hydrologie, Hydrogeologie und Geologie. Beiträge zur COG Fachtagung Salzburg., S. 169-188 (2007)

Carlé, W.: Die Mineral- und Thermalwässer Mitteleuropas - Geologie Chemismus Genese. Wissenschaftliche Verlagsgesellschaft $\mathrm{mbH}$, Stuttgart (1975)

Chalikakis, K., Plagnes, V., Guerin, R., Valois, R., Bosch, F.P.: Contribution of geophysical methods to karst-system exploration: an overview. Hydrogeol. J. 19(6), 1169 (2011)

Clark, I.D., Fritz, P.: Environmental isotopes in hydrogeology. CRC press, London, New York (2013)

Cliff, R.A., Spötl, C., Mangini, A.: U-Pb dating of speleothems from Spannagel Cave, Austrian Alps: a high resolution comparison with U-series ages. Quat. Geochronol. 5(4), 452-458 (2010)

Czurda, K.: Die Akratothermen von Hintertux. Die Mineral- und Heilquellen Österreichs, S. 94-95 (1993)

D’Amore, F., Arnórsson, S.: Geothermometry. In: Arnórsson, S. (ed.), Isotopic and chemical techniques in geothermal exploration, development and use. Sampling methods, data handling, interpretation. International Atomic Energy Agency, Vienna, pp. 152-199 (2000)

Elster, D., Fischer, L., Hann, S., Goldbrunner, J., Schubert, G., Berka, R., Hobiger, G., Legerer, P., Philippitsch, R.: Österreichs Mineralund Heilwässer. Geologische Bundesanstalt, Wien, S. 450 (2018)

Fecht, M., Höfle, B., Starnberger, R., Kaser, G.: Eine Karte der aktuellen Verdunstung für das Tirol Atlas Gebiet anhand von Landnutzungs- und Vegetationsdaten, Institut für Geographie, University of Innsbruck, 1-26, 2005. University of Innsbruck (2009). http:// tirolatlas.uibk.ac.at/topics/water/pub/evaporation.pdf. Zugegriffen: 10. Feb. 2018

Finger, F., Frasl, G., Haunschmid, B., Lettner, H., von Quadt, A., Schermaier, A., Schindlmayr, A.O., Steyrer, H.P.: The Zentralgneise of the Tauern Window (eastern Alps): insight into an intraAlpine Variscan batholith. In: Pre-Mesozoic Geology in the Alps, S. 375-391. Springer, Berlin (1993)

Fischer, A., Seiser, B., Stocker Waldhuber, M., Mitterer, C., Abermann, J.: Tracing glacier changes in Austria from the Little Ice Age to the present using a lidar-based high-resolution glacier inventory in Austria. Cryosphere, 9(2) (2015). https://doi.org/10.5194/tc-9753-2015

Frisch, W.: Die stratigraphisch-tektonische Gliederung der Schieferhülle und die Entwicklung des penninischen Raumes im westlichen Tauernfenster (Gebiet Brenner-Gerlospaß). Mitt. Geol. Ges. Wien 66(67), 9-20 (1974)

Frisch, W., Neubauer, F., Satir, M.: Concepts of the evolution of the Austroalpine basement complex (Eastern Alps) during the Caledonian-Variscan cycle. Geol. Rundsch. 73, 47-68 (1984)

Fügenschuh, B., Mancktelow, N.S., Schmid, S.S.: Comment on Rosenberg and Garcia: estimating displacement along the Brenner Fault and orogen-parallel extension in the Eastern Alps, Int. J. Earth Sci. (Geol Rundsch) (2011) 100, 1129-1145. Int. J. Earth. Sci. 101(5), 1451-1455 (2012)

Geologische Bundesanstalt (GBA): GEOFAST 1: 50 000. Geologische Bundesanstalt (GBA), Lanersbach (2011). Blatt 149

Giggenbach, W.F., Goguel, R.L.: Methods for the collection and analysis of geothermal and volcanic water and gas samples. Chemistry Division, Department of Scientific and Industrial Research, Petone (1988)

Giggenbach, W.F.: Chemical techniques in geothermal exploration. In: D'Amore, F. (ed.) Application of Geochemistry in Geothermal Reservoir Development, UNITAR/UNDP Centre on small energy resources, Rome, 119-144 (1991)

Glodny, J., Ring, U., Kühn, A.: Coeval high—pressure metamorphism, thrusting, strike-slip, and extensional shearing in the Tauern Window, Eastern Alps. Tectonics 27(4), 1-27 (2008)

Goldbrunner, J.: Austria-country update. In: Proceedings World Geothermal Congress, S. 1-13. (2010)

Götzl, G., Ostermann, V., Kalasek, R., Heimrath, R., Haindlmaier, G., Novak, A., Zottl, A.: Seichtes Geothermie Potenzial Österreichs. Überregionale, interdisziplinäre Potenzialstudie zur Erhebung und Darstellung des oberflächennahen geothermischen Anwendungspotenzials auf Grundlage eines regelmäßigen Bearbeitungsrasters. Österr. Abfall Wasserwirtsch. (2010). https://doi. org/10.1007/s00506-010-0185-2

Heldmann, C.-D., Sass, I., Schäffer, R.: A persistent local thermal anomaly in the Ahorn gneiss recharged by glacier melt water (Austria). Hydrogeol J (2019). https://doi.org/10.1007/s10040019-02034-8

Hilberg, S.: Natural tracers in fractured hard-rock aquifers in the Austrian part of the Eastern Alps-previous approaches and future perspectives for hydrogeology in mountain regions. Hydrogeol. J. 24, 1091-1105 (2016)

IAEA, WMO: Global network of isotopes in precipitation: the GNIP database (2018). http://www.iaea.org/water. Zugegriffen: 19. Mai 2018

Jansson, P., Hock, R., Schneider, T.: The concept of glacier storage: a review. J. Hydrol. Reg. Stud. 282(1-4), 116-129 (2003)

Kampschulte, A., Strauss, H.: The sulfur isotopic evolution of Phanerozoic seawater based on the analysis of structurally substituted sulfate in carbonates. Chem. Geol. 204(3-4), 255-286 (2004)

Kiessling, W.: Palaeontological and facial features of the Upper Jurassic Hochstegen marble (Tauern window, Eastern Alps). Terra Nova 4(2), 184-197 (1992)

Kilchmann, S., Waber, H.N., Parriaux, A., Bensimon, M.: Natural tracers in recent groundwaters from different Alpine aquifers. Hydrogeol. J. 12(6), 643-661 (2004)

Krainer, K., Mostler, W., Spötl, C.: Discharge from active rock glaciers, Austrian Alps: a stable isotope approach. Austrian J. Earth Sci. 100, 102-112 (2007)

Kralik, M., Papesch, W., Stichler, W.: Austrian Network of Isotopes in Precipitation (ANIP): Quality assurance and climatological phenomenon in one of the oldest and densest networks in the world. In: Isotope Hydrology and Integrated Water Resources Management, IAEA-CSP-23, 146-149 (2003). http://citeseerx. ist.psu.edu/viewdoc/download?doi=10.1.1.362.846\&rep=rep1\& type $=$ pdf\#page $=162$ 
Kurz, W., Neubauer, F., Genser, J., Dachs, E.: Alpine geodynamic evolution of passive and active continental margin sequences in the Tauern Window (eastern Alps, Austria, Italy): a review. Geol. Rundsch. 87, 225-242 (1998)

Kurz, W., Neubauer, F., Unzog, W., Genser, J., Wang, X.: Microstructural and textural development of calcite marbles during polyphase deformation of Penninic units within the Tauern Window (Eastern Alps). Tectonophysics 316, 327-342 (2000). https://doi.org/ 10.1016/S0040-1951(99)00257-7

Lammerer, B., Gebrande, H., Lüschen, E., Veselá, P.: A crustal-scale cross-section through the Tauern Window (eastern Alps) from geophysical and geological data. Special Publications 298. Geological Society, London, S. 219-229 (2008) https://doi.org/10. 1144/SP298.11

Langguth, H.R., Voigt, R.: Hydrogeologische Methoden. Springer, Heidelberg Berlin (2013)

Ledoux, H.: Paläogeographie und tektonische Entwicklung im Penninikum des Tauern-Nordwestrandes im oberen Tuxer Tal. Jahrb. Geol. B. A 126, 359-368 (1984)

Lund, J.W.: Direct utilization of geothermal energy. Energies 3(8), 1443-1471 (2010). https://doi.org/10.3390/en3081443

Markart, G., Kohl, B., Sotier, B., Klebinder, K., Schauer, T., Bunza, G., Pirkl, H., Stern, R.: A simple code of practice for the assessment of surface runoff coefficients for alpine soil-/vegetation units in torrential rain (version 2.0). Natural Hazards and Landscaper (BFW), Innsbruck (2011)

Markart, G., Römer, A., Bieber, G., Pirkl, H., Klebinder, K., Hörfarter, C., Ita, A., Jochum, B., Kohl, B., Motschka, K.: Assessment of shallow interflow velocities in Alpine catchments for the improvement of hydrological modelling. Eng. Geol. Soc. Territ. 3, 611-615 (2015)

Moser, H., Rauert, W., Behrens, H.: Isotopenmethoden in der Hydrologie. Gebr. Bornträger, Berlin, Stuttgart (1980)

Neubarth, J., Kaltschmitt, M. (Hrsg.): Erneuerbare Energien in Österreich: Systemtechnik, Potenziale, Wirtschaftlichkeit, Umweltaspekte. Springer, Berlin (2013)

Neubauer, F., Genser, J., Kurz, W., Wang, X.: Exhumation of the Tauern window, Eastern Alps. Phys. Chem. Earth Part A: Solid Earth Geod. 24, 675-680 (1999). https://doi.org/10.1016/S14641895(99)00098-8

Parkhurst, D.L., Appelo, C.A.J.: Description of input and examples for PHREEQC version 3: a computer program for speciation, batchreaction, one-dimensional transport, and inverse geochemical calculations (Report No. 6-A43). US Geological Survey, Denver (2013)

Paul, F., Frey, H., Le Bris, R.: A new glacier inventory for the European Alps from Landsat TM scenes of 2003: challenges and results. Ann. Glaciol. 52(59), 144-152 (2011)

Powell, T., Cumming, W.: Spreadsheets for geothermal water and gas geochemistry. In: Proceedings thirty-fifth workshop on geothermal reservoir engineering, S. 1-3. (2010)

Rimstidt, J.D.: Quartz solubility at low temperatures. Geochim. Cosmochim. Acta 61(13), 2553-2558 (1997)

Rosenberg, C.L., Berger, A.: On the causes and modes of exhumation and lateral growth of the Alps. Tectonics 28(6), 1-16 (2009)

Rosenberg, C.L., Garcia, S.: Estimating displacement along the Brenner Fault and orogen-parallel extension in the Eastern Alps. Int. J. Earth Sci. 100(5), 1129-1145 (2011)

Rosenberg, C.L., Garcia, S.: Reply to the comment of Fügenschuh et al on the paper 'Estimating displacement along the Brenner Fault and orogen-parallel extension in the Eastern Alps' by Rosenberg and Garcia, Int. J. Earth Sci. (Geol. Rundsch.) (2011) 100, 1129-1145. Int. J. Earth Sci. 101(5), 1457-1464 (2012)

Rosenberg, C.L., Schneider, S.: The western termination of the SEMP fault (Eastern Alps) and its bearing on the exhumation of the Tauern Window. Geol. Soc. Lond. Special Publ. 298(1), 197-218 (2008). https://doi.org/10.1144/SP298.10
Sass, I., Heldmann, C.-D., Lehr, C.: Erschließung eines Marmorkarstvorkommens als mitteltiefer Erdwärmesondenspeicher im Tuxertal, Tirol. Grundwasser 21(2), 137-145 (2016a)

Sass, I., Heldmann, C.-D., Schäffer, R.: Erkundung und Beweissicherung für eine geothermale Erschließung eines Alpinen Karstaquifers im Tuxertal, Österreich. Grundwasser 21(2), 147-156 (2016b). https://doi.org/10.1007/s00767-015-0312-x

Schäffer, R., Sass, I.: Ergebnisse von Markierungsversuchen am Grinberg, Tuxertal, Österreich. In: Banning, A., Frank, S., KaufmannKnoke, R., Niedermayr, A., Wisotzky, F., Wohnlich, S. (Hrsg.) 26. Fachtagung der Fachsektion Hydrogeologie e. V. in der DGGV e. V., Bochum, 21.-24. März 2018. Bochumer Geowissenschaftliche Arbeiten, Bd. 24. (2018)

Schäffer, R., Sass, I., Heldmann, C.-D., Hesse, J.C., Hintze, M., Scheuvens, D., Schubert, G., Seehaus, R.: Wasserwirtschaftliche Schlussfolgerungen aus der Stichtagsbeprobung eines $225 \mathrm{~km}^{2}$ großen Einzugsgebietes im NW Tauernfenster, Österreich. Grundwasser 25(1), 53-68 (2020). https://doi.org/10. 1007/s00767-019-00436-9

Schmid, S.M., Scharf, A., Handy, M.R., Rosenberg, C.L.: The Tauern Window (Eastern Alps, Austria): A new tectonic map, with crosssections and a tectonometamorphic synthesis. Swiss J. Geosci. 106, 1-32 (2013). https://doi.org/10.1007/s00015-013-0123-y

Schneider, S.: Exhumation mechanisms of middle and lower crust in the western Tauern Window, Eastern Alps. Doctoral thesis. Freie Universität Berlin, Berlin (2014)

Schneider, S., Hammerschmidt, K., Rosenberg, C.L.: Dating the longevity of ductile shear zones: Insight from 40Ar/39Ar in situ analyses. Earth Planet. Sci. Lett. 369-370, 43-58 (2013). https://doi. org/10.1016/j.eps1.2013.03.002

Schön, C., Lammerer, B.: Strainanalyse an grobklastischen Metasedimenten des westlichen Tauernfensters. Abh Geol B-A 49, 97-106 (1990)

Selverstone, J.: Evidence for east-west crustal extension in the Eastern Alps: Implications for the unroofing history of the Tauern Window. Tectonics 7(1), 87-105 (1988)

Spötl, C., Mangini, A.: Paleohydrology of a high-elevation, glacierinfluenced karst system in the Central Alps (Austria). Austrian J. Earth Sci. 103(2), 92-105 (2010)

Staudt, W.J., Schoonen, M.A.A.: Sulfate incorporation into sedimentary carbonates. In: Vairavamurthy, A., Schoonen, M.A.A. (Hrsg.) Geochemical Transformation of Sedimentary Sulfur Amer. Chem. Soc. Symp. Ser. 612. S. 323-345. (1995)

Strauss, H.: The isotopic composition of sedimentary sulfur through time. Palaeogeogr. Palaeoclimatol. Palaeoecol. 132(1-4), 97-118 (1997)

Thiele, O.: Zur Stratigraphie und Tektonik der Schieferhülle der westlichen Hohen Tauern. Verhandlungen der geologischen Bundesanstalt. Geologische Bundesanstalt, Wien, S. 230-244 (1970)

Töchterle, A., Brandner, R., Reiter, F.: Strain partitioning on major fault zones in the north western Tauern Window-Insights from the investigations to the Brenner Base Tunnel. Austrian J. Earth Sci. 104(1), 15-35 (2011)

VBI, Verband Beratender Ingenieure: VBI-Leitfaden Oberflächennahe Geothermie, 3. Aufl. Schriftenreihe der VBI, Bd. 18. (2012)

VBI, Verband Beratender Ingenieure: VBI-Leitfaden Tiefe Geothermie, 2. Aufl. Schriftenreihe der VBI, Bd. 21. (2013)

Verma, M.P.: Revised quartz solubility temperature dependence equation along the water-vapor saturation curve. In: World Geothermal Congress Kyushu-Tohoku. S. 1927-1932. (2000)

Vosteen, H.D., Rath, V., Clauser, C., Lammerer, B.: The thermal regime of the Eastern Alps from inversion analyses along the TRANSALP profile. Phys. Chem. Earth Parts A/B/C 28(9-11), 393-405 (2003)

Winkler, G., Wagner, T., Pauritsch, M., Birk, S., Kellerer-Pirklbauer, A., Benischke, R., Leis, A., Morawetz, R., Schreilechner, M.G., Hergarten, S.: Identification and assessment of groundwater flow and storage components of the relict Schöneben Rock Glacier, 
Niedere Tauern Range, Eastern Alps (Austria). Hydrogeol. J. 24(4), 937-953 (2016)

Wisotzky, F., Cremer, N., Lenk, S.: Angewandte Grundwasserchemie,

Hydrogeologie und Hydrogeochemische Modellierung: Grundlagen, Anwendungen und Problemlösungen. Springer, Berlin, Heidelberg (2018)
Zötl, J., Goldbrunner, J. (Hrsg.): Die Mineral- und Heilwässer Österreichs: geologische Grundlagen und Spurenelemente. Springer, Berlin Heidelberg (2013)

Hinweis des Verlags Der Verlag bleibt in Hinblick auf geografische Zuordnungen und Gebietsbezeichnungen in veröffentlichten Karten und Institutsadressen neutral. 